\title{
THE CLASSIFICATION OF BIRTH AND DEATH PROCESSES
}

\author{
BY \\ SAMUEL KARLIN AND JAMES MCGREGOR
}

The transition probability matrix $P(t)=\left(P_{i j}(t)\right)$,

$$
P_{i j}(t)=\operatorname{Pr}\{X(t+s)=j \mid X(s)=i\}, \quad i, j=0,1,2, \cdots ; t, s \geqq 0
$$

of a birth and death process $X(t)$ satisfies the differential equations

$$
\begin{aligned}
& P^{\prime}(t)=A P(t), \quad(t \geqq 0), \\
& P^{\prime}(t)=P(t) A,
\end{aligned}
$$

the initial condition

$$
P(0)=I,
$$

and has the additional properties

$$
\begin{aligned}
P_{i j}(t) & \geqq 0, \\
\sum_{j=0}^{\infty} P_{i j}(t) & \leqq 1, \\
P(t+s) & =P(t) P(s) .
\end{aligned}
$$

The matrix $A=\left(a_{i j}\right)$ in $(0.1)$ is of the form

$$
\begin{aligned}
a_{i, i+1} & =\lambda_{i}, \\
a_{i, i} & =-\left(\lambda_{i}+\mu_{i}\right), \\
a_{i, i-1} & =\mu_{i}, \\
a_{i j} & =0 \text { if }|i-j|>1,
\end{aligned}
$$

where $\lambda_{i}>0$ for $i \geqq 0, \mu_{i}>0$ for $i \geqq 1$, and $\mu_{0} \geqq 0$.

In the applications one is given the matrix $A$ and it is required to construct $P(t)$ and to study the properties of the corresponding stochastic process. The existence, uniqueness, and the analytic properties of $P(t)$ have been discussed in detail in [1]. The objective of this paper is to use the results of [1] to establish equivalences between properties of the stochastic process and properties of the sequences $\left\{\lambda_{n}\right\},\left\{\mu_{n}\right\}$, and to evaluate, in terms of these sequences, some of the interesting probabilistic quantities associated with the process.

Received by the editors August 2, 1956. 
It is assumed throughout that the state space of the process $X(t)$ is the set of all non-negative integers. Processes with only a finite number of states can be treated by the same methods.

In $[1$, Theorems 14,15$]$ it is shown that there is one and only one matrix $P(t)$ which satisfies $(0.1),(0.2),(0.3),(0.4),(0.5)$ if and only if

$$
\sum_{n=0}^{\infty}\left(\pi_{n}+\frac{1}{\lambda_{n} \pi_{n}}\right)=\infty,
$$

where

$$
\pi_{0}=1, \quad \pi_{n}=\frac{\lambda_{0} \lambda_{1} \cdots \lambda_{n-1}}{\mu_{1} \mu_{2} \cdots \mu_{n}} \quad \text { for } n \geqq 1 .
$$

Since we know of no application in which $(0.7)$ is not satisfied, it will always be assumed that $(0.7)$ is valid.

Associated with the matrix $A$ is a system $\left\{Q_{n}(x)\right\}$ of polynomials defined by the recurrence formulas

$$
\begin{aligned}
Q_{0}(x) & =1, \\
-x Q_{0}(x) & =-\left(\lambda_{0}+\mu_{0}\right) Q_{0}(x)+\lambda_{0} Q_{1}(x), \\
-x Q_{n}(x) & =\mu_{n} Q_{n-1}(x)-\left(\lambda_{n}+\mu_{n}\right) Q_{n}(x)+\lambda_{n} Q_{n+1}(x), \quad n \geqq 1 .
\end{aligned}
$$

It is shown in [1] that there is at least one positive regular measure $\psi$ on $0 \leqq x<\infty$ such that

$$
\int_{0}^{\infty} Q_{i}(x) Q_{j}(x) d \psi(x)=\frac{\delta_{i j}}{\pi_{j}}, \quad i, j=0,1,2, \cdots .
$$

Any suth measure $\psi$ is called a solution of the moment problem. We are going to assume throughout that the solution of the moment problem is unique. If $\mu_{0}=0$ this is equivalent to condition (0.7). If $\mu_{0}>0$ the solution of the moment problem is unique if and only if

$$
\sum_{n=0}^{\infty} \pi_{n}\left(1+\mu_{0} \sum_{k=0}^{n-1} \frac{1}{\lambda_{k} \pi_{k}}\right)^{2}=\infty,
$$

and looking at the term $2 \mu_{0} \pi_{n} / \lambda_{n-1} \pi_{n-1}=2 \mu_{0} / \mu_{n}$, it is seen that a sufficient condition for $(0.11)$ is

$$
\sum_{n=1}^{\infty} \frac{1}{\mu_{n}}=\infty .
$$

This last condition is satisfied in all practical applications.

The transition matrix $P(t)$ is represented by the formula

$$
P_{i j}(t)=\pi_{j} \int_{0}^{\infty} e^{-x t} Q_{i}(x) Q_{j}(x) d \psi(x)
$$


The usefulness of this integral representation derives from the simple monotonic properties of $e^{-x t}$, and from the fact that the dependence on $t, i$ and $j$ is factored in the integrand.

In the first four sections the behavior of the process as regards ergodicity, recurrence, and transience is characterized in terms of the behavior of the sequences $\left\{\pi_{n}\right\},\left\{1 / \lambda_{n} \pi_{n}\right\}$. In these sections the first passage time distributions for the ergodic case are studied and the problem of existence and computation of the moments of these distributions is reduced to the existence and computation of integrals of the form

$$
\int_{0+}^{\infty} \frac{d \psi(x)}{x^{k}}
$$

The evaluation of these integrals in terms of the $\pi_{n}$ and $1 / \lambda_{n} \pi_{n}$ is carried out in the appendix. In the transient case the distribution of the "time at which $\infty$ is reached" is examined, and the moments of this distribution are expressed in terms of integrals of the form

$$
\int_{0}^{\infty} \frac{d \psi}{x^{k}}
$$

In $\S 5$ the case where absorption occurs from the zero state into an ignored minus-one state $\left(\mu_{0}>0\right)$ is treated. The moments of the absorption time are computed and similar calculations are made for the distribution of the number of transitions before absorption occurs.

$\S 6$ discusses some general relations between absorbing and nonabsorbing processes.

In $\$ 7$ a very strong ergodic theorem for birth and death processes is given. It is a Tauberian form of the classical Doeblin ratio theorem.

$\S 8$ treats the problem of computing $\psi$ when the matrix $A$ is given.

In another publication the results of this paper will be applied to the study of linear growth and certain queuing models.

1. Recurrence and ergodicity $\left(\mu_{0}=0\right)$. From the integral representation (0.12) it follows that $\lim _{t \rightarrow \infty} P_{i j}(t, \psi)$ exists and, since all $Q_{n}(0)=1$, is equal to $\pi_{j} \rho$ where $\rho$ is the mass of $\psi$ at $x=0$. The constant $\rho$ is given by

$$
\rho=1 / \sum_{n=0}^{\infty} \pi_{n}
$$

which is to be interpreted as zero if the series diverges. Thus the classical ergodic theorem concerning the behavior of $P_{i j}(t)$ as $t \rightarrow \infty$ is a trivial consequence of the integral representation. It is also easy to see that if $\psi$ has no mass near $x=0$ except at $x=0$, then $P_{i}(t)$ converges exponentially to its limiting value. 
We now turn to the study of recurrence properties. The recurrence time distributions $F_{i i}(t)$ and first passage time distributions $F_{i j}(t)$ are defined by (i) $F_{i j}(t)=\operatorname{Pr}\{X(\tau)=j$ for some $\tau, 0<\tau \leqq t \mid X(0)=i\}, \quad i \neq j$, (ii) $F_{i i}(t)=\operatorname{Pr}\left\{X\left(\tau_{1}\right) \neq i, X\left(\tau_{2}\right)=i\right.$ for some $\left.\tau_{1}, \tau_{2}, 0<\tau_{1}<\tau_{2} \leqq t \mid X(0)=i\right\}$. In words, $F_{i j}(t), i \neq j$, is the probability that the particle, having started at $i$, visits $j$ some time before $t$, and $F_{i i}(t)$ is the probability that the particle, having started at $i$, leaves $i$ and then returns to $i$ some time before $t$. A rigorous discussion of these quantities has been given by Chung [2]. It may happen that some of these "distributions" are not honest probability distributions, i.e.,

$$
\int_{0}^{\infty} d F_{i i}(t)<1, \quad \int_{0}^{\infty} d F_{i j}(t)<1 .
$$

The integral $\int_{0}^{\infty} d F_{i i}(t)$ is the probability that if the particle starts at $i$, it leaves $i$ and then returns to $i$ in finite time. The $i$ th state is called a recurrent state if $\int_{0}^{\infty} d F_{i i}(t)=1$, and is called a transient state otherwise. A recurrent state $i$ is called ergodic or recurrent null according as its expected recurrence time $\int_{0}^{\infty} t d F_{i i}(t)$ is finite or infinite. A process is called recurrent, ergodic, recurrent null, or transient if every one of its states has the corresponding property.

It is clear that

$$
\operatorname{Pr}\{X(s+\tau)=i \text { for all } \tau, 0 \leqq \tau \leqq t \mid X(s)=i\}=\exp \left(-\left(\lambda_{i}+\mu_{i}\right) t\right) .
$$

By a standard enumeration of paths it is found that

$$
\begin{aligned}
& P_{i i}(t)=\exp \left(-\left(\lambda_{i}+\mu_{i}\right) t\right)+\int_{0}^{t} P_{i i}(t-s) d F_{i i}(s), \\
& P_{i j}(t)=\int_{0}^{t} P_{j j}(t-s) d F_{i j}(s) .
\end{aligned}
$$

Introducing the Laplace transforms,

$$
\hat{P}_{i j}(s)=\int_{0}^{\infty} e^{-s t} P_{i j}(t) d t \quad \text { and } \quad \hat{F}_{i j}(s)=\int_{0}^{\infty} e^{-s t} d F_{i j}(t)
$$

we obtain from (1.1)

$$
\hat{P}_{i i}(s)=\frac{1}{\left(q_{i}+s\right)\left(1-\hat{F}_{i i}(s)\right)} \quad \text { and } \quad \hat{P}_{i j}(s)=\hat{P}_{j j}(s) \hat{F}_{i j}(s)
$$

where $q_{i}=\lambda_{i}+\mu_{i}$. As $s \rightarrow 0, \hat{F}_{i i}(s) \rightarrow \int_{0}^{\infty} d F_{i i}(t) \leqq 1$ so that the recurrence probabilities define true distribution functions if and only if $\hat{P}_{i i}(s)$ tends to $\infty$ or equivalently $\int_{0}^{\infty} P_{i i}(t)=\infty$. This last condition by integration of $(0.12)$ reduces to 


$$
\int_{0}^{\infty} \frac{Q_{i}^{2}(x)}{x} d \psi=\infty .
$$

Thus the amount of mass of $\psi$ in the neighborhood of the origin can be thought of as a measure of recurrence for the process. Since $Q_{i}(0)=1$ for all $i$, evidently $\int Q_{i}^{2}(x) d \psi / x=\infty$ for any $i$ if and only if $\int d \psi / x=\infty$. The classical result that either all states or no states are recurrent is now apparent. Furthermore, from (1.3) it follows that if $\int d \psi / x=\infty$, then

$$
\lim _{s \rightarrow 0} \hat{F}_{i j}(s)=\lim _{s \rightarrow 0}\left(\int \frac{Q_{i}(x) Q_{j}(x) d \psi}{x+s} / \int \frac{Q_{j}^{2}(x) d \psi}{x+s}\right)=1 .
$$

Hence, if the process is recurrent then $\int_{0}^{\infty} d F_{i j}(t)=1$. Because of the relation $\int d \psi / x=\sum 1 / \lambda_{n} \pi_{n}$ (see (9.9)), we obtain the following useful recurrence criterion.

THEOREM 1. The process is recurrent if and only if $\sum 1 / \lambda_{n} \pi_{n}=\infty$.

This last condition is easy to check since the process is usually defined in terms of the birth and death rates $\lambda_{n}$ and $\mu_{n}$.

It follows from Theorem 1 that if the process is recurrent, then the spectrum of $\psi$ reaches to the origin.

In the remainder of this section we restrict our attention exclusively to the zero state. All the results remain valid, after minor modifications, for any state in the process.

THEOREM 2. (a) The process is ergodic if and only if $\sum \pi_{n}<\infty$ and $\sum 1 / \lambda_{n} \pi_{n}$ $=\infty$.

(b) The process is null recurrent if and only if $\sum \pi_{n}=\infty$ and $\sum 1 / \lambda_{n} \pi_{n}=\infty$.

Proof. It is known that it is sufficient to verify the statements of the theorem for the zero state. If $\sum \pi_{n}<\infty$ and $\sum 1 / \lambda_{n} \pi_{n}=\infty$, then $\psi$ has a mass at zero equal to $1 / \sum \pi_{n}=\rho$ and $\lim _{t \rightarrow \infty} P_{i j}(t)=\rho \pi_{j}$. Computing the first moment of $F_{00}$, we get

$$
-\hat{F}_{00}^{\prime}(s)=\frac{-\hat{P}_{00}^{\prime}(s)}{\left(q_{0}+s\right) \hat{P}_{00}^{2}(s)}-\frac{1}{\left(q_{0}+s\right)^{2}} \frac{1}{\hat{P}_{00}(s)} .
$$

The second term tends to zero as $s \rightarrow 0$ since $\hat{P}_{00}(s) \rightarrow \infty$. Observe that

$$
\frac{-\hat{P}_{00}^{\prime}(s)}{\hat{P}_{00}^{2}(s)}=\frac{\int \frac{d \psi}{(x+s)^{2}}}{\left(\int \frac{d \psi}{(x+s)}\right)^{2}}=\frac{\left(\frac{\rho}{s^{2}}+\int \frac{d \psi^{*}}{(x+s)^{2}}\right)}{\left(\frac{\rho}{s}+\int \frac{d \psi^{*}}{(x+s)}\right)^{2}}
$$


where $\psi^{*}$ is obtained from $\psi$ by removing the mass $\rho$ at 0 , so that $\psi^{*}$ becomes continuous at 0 . It is easy to see upon multiplying numerator and denominator by $s^{2}$ that $\lim _{s \rightarrow 0}-\hat{F}_{00}^{\prime}(s)=1 / q_{0} \rho=\int_{0}^{\infty} t d F_{00}(t)$. Conversely, if the zero state is ergodic, then the irreducible $a$-periodic Markov chain $P_{i i}(n h)$ where $h$ is fixed is ergodic. By appealing to the theory of Markov chains, we deduce that $\lim _{n \rightarrow \infty} P_{i i}(n h)$ is positive so that $\rho>0$. But, $\rho=1 / \sum \pi_{i}$ and hence $\sum \pi_{i}<\infty$.

The conclusion of (b) is immediate from (a).

2. Some simple recurrence criteria $\left(\mu_{0}=0\right)$. In this section some additional methods of testing for recurrence are developed. The results here are motivated by some theorems of Foster [3].

LEMma 1. The process is transient if and only if there is a nonconstant bounded vector $\bar{c}=\left\{c_{0}, c_{1}, \cdots\right\}$ such that

$$
A \bar{c}=0 \text { except for the first component. }
$$

Proof. If the process is transient then

$$
c_{0}^{*}=0, \quad c_{n}^{*}=\sum_{i=0}^{n-1} \frac{1}{\lambda_{i} \pi_{i}},
$$$$
n \geqq 1,
$$

defines a nonconstant bounded vector $\bar{c}^{*}$ which satisfies the condition.

Conversely, if $\bar{c}$ is such a vector then, $\bar{c}$ is nonconstant, the first component of $A \bar{c}^{*}$ is not zero. Hence for suitable $\alpha \neq 0$

$$
\bar{e}=\bar{c}-\alpha \bar{c}^{*}
$$

is a solution of $A \bar{e}=0$. Consequently $\bar{e}$ is a constant vector and $c^{*}$ is bounded. Thus $\sum_{0}^{\infty} 1 / \lambda_{n} \pi_{n}$ converges and the process is transient.

THEOREM 3. The process is recurrent if and only if there is an unbounded vector $\bar{d}=\left\{d_{n}\right\}$ such that $d_{n} \rightarrow+\infty$ and $A \bar{d} \leqq 0$ except for the first component.

Proof. Define $\bar{c}^{*}$ as in the lemma and let $\bar{e}=\{1,1, \cdots, 1, \cdots\}$. If the process is recurrent then $\bar{c}^{*}$ is unbounded, $c_{n}^{*} \rightarrow+\infty$ and $A \bar{c}^{*} \leqq 0$ except for the first component.

Suppose there is a vector $\bar{d}$ satisfying the conditions of the lemma. Choose $\alpha$ and $\beta$ so that the first two components of the vector

$$
\bar{f}=\bar{d}-\alpha \bar{e}-\beta \bar{c}^{*}
$$

are both zero. Then $A \bar{f}=A \bar{d} \leqq 0$ for all components beyond the first, and the first component of $A \bar{f}$ is zero. From $f_{0}=f_{1}=0$ and

$$
\lambda_{n}\left(f_{n+1}-f_{n}\right)=(A \bar{f})_{n}+\mu_{n}\left(f_{n}-f_{n-1}\right), \quad n \geqq 1,
$$

it follows by induction that

$$
0=f_{1} \geqq f_{2} \geqq f_{3} \geqq \cdots \geqq f_{n} \geqq \cdots .
$$

Since $d_{n} \rightarrow+\infty$ and $e_{n}=1$ for all $n$ it is seen that $c_{n}^{*} \rightarrow+\infty$, which proves the process recurrent. 
It may be remarked in passing that the sequence $\pi=\left\{\pi_{n}\right\}$ is to within a constant factor the only solution of $\varepsilon A=0$.

3. Moments of the first passage time distributions $\left(\mu_{0}=0\right)$. The object of this section is to compute the moments of the first passage time distributions $F_{i j}(t)$ in terms of the constants $\pi_{n}$ and $1 / \lambda_{n} \pi_{n}$. The details of the computation are given only in the case of $F_{i i}(t)$. It is assumed throughout that $\sum \pi_{n}<\infty$, $\sum 1 / \lambda_{n} \pi_{n}=\infty$, these being the necessary and sufficient conditions for the first moments of the $F_{i j}(t)$ to be finite.

The measure $\psi$ has mass $\rho=1 / \sum \pi_{n}$ at $x=0$. Let $\psi^{*}$ be the measure obtained from $\psi$ by removing the mass at $x=0$. Choose some fixed $i$ and let

$$
I_{k}=\int_{0}^{\infty} Q_{i}^{2}(x) \frac{d \psi^{*}(x)}{x^{k}} \leqq \infty, \quad k=1,2, \cdots .
$$

From (1.3) it follows that for $s>0$

$$
\begin{aligned}
1-\int_{0}^{\infty} e^{-s t} d F_{i i}(t) & =\frac{1}{\left(q_{i}+s\right)\left(\frac{\rho \pi_{i}}{s}+\pi_{i} \int_{0}^{\infty} Q_{i}^{2}(x) \frac{d \iota^{*}}{x+s}\right)} \\
& =\frac{s}{\rho q_{i} \pi_{i}} \cdot \frac{1}{1+s / q_{i}}\left[1+\frac{1}{\rho} \int_{0}^{\infty} \frac{Q_{i}^{2}(x) s d \psi^{*}}{x+s}\right]^{-1}
\end{aligned}
$$

where $q_{i}=\lambda_{i}+\mu_{i}$. Since $s /(x+s) \rightarrow 0$ boundedly on $0<x<\infty$ as $s \rightarrow 0$

$$
\left[1+\frac{1}{\rho} \int_{0}^{\infty} \frac{Q_{i}^{2}(x) s}{x+s} d \psi^{*}\right]^{-1}=\sum_{n=0}^{\infty}\left(-\frac{1}{\rho} \int_{0}^{\infty} \frac{Q_{i}^{2}(x) s d \psi^{*}}{x+s}\right)^{n}
$$

for small $s>0$.

Now suppose $I_{k}<\infty$. Then

$$
\frac{1}{\rho} \int_{0}^{\infty} \frac{Q_{i}^{2}(x) s d \psi^{*}}{x+s}=\frac{-1}{\rho}\left[\sum_{l=1}^{k}(-s)^{l} I_{l}+(-1)^{k} s^{k} J_{k}\right]
$$

where

$$
J_{k}=\int_{0}^{\infty} \frac{Q_{i}^{2}(x)}{x^{k}} \frac{s}{x+s} d \psi^{*}(x) \rightarrow 0 \quad \text { as } s \rightarrow 0 .
$$

Hence

$$
\begin{aligned}
{\left[1+\frac{1}{\rho} \int_{0}^{\infty} \frac{Q_{i}^{2}(x) s}{x+s} d \psi^{*}\right]^{-1} } & =\sum_{n=0}^{k}\left(\frac{1}{\rho} \sum_{l=1}^{k}(-1)^{l} s^{l} I_{l}\right)^{n}+o\left(s^{k}\right) \\
& =\sum_{p=0}^{k}(-s)^{p} c_{p}+o\left(s^{k}\right)
\end{aligned}
$$


where $c_{0}=1$ and for $p>0$

$$
c_{p}=\sum_{\nu_{1}+2 \nu_{2}+\ldots+k v_{k}=p}(\rho)^{-\left(\nu_{1}+\cdots+\nu_{k}\right)} \frac{\left(\nu_{1}+\cdots+\nu_{k}\right) !}{\nu_{1} ! \cdots \nu_{k} !} I_{1}^{\nu_{1}} \cdots I_{k}^{\nu_{k}} .
$$

Consequently,

$$
1-\int_{0}^{\infty} e^{-s t} d F_{i i}(t)=\frac{s}{\rho q_{i} \pi_{i}} \sum_{p=0}^{k}(-s)^{p} \sum_{r=0}^{p} c_{p-r} \frac{(-1)^{r}}{q_{i}^{r}}+o\left(s^{k+1}\right)
$$

from which it follows that $\int_{0}^{\infty} t^{k+1} d F_{i i}(t)<\infty$. Using an induction argument it is not difficult to show that conversely if $\int_{0}^{\infty} t^{k+1} d F_{i i}(t)<\infty$ then $I_{k}<\infty$. This gives the following:

THEOREM 4. The $(k+1)$ st moment of $F_{i i}(t)$ is finite if and only if $\int_{0}^{\infty} d \psi^{*}(x) / x^{k}$ $<\infty$, in which case

$$
a_{k+1}=\int_{0}^{\infty} t^{k+1} d F_{i i}(t)=\frac{(k+1) !}{\rho q_{i} \pi_{i}} \sum_{n=0}^{k} \frac{1}{q_{i}^{n}} c_{k-n} .
$$

The integrals $I_{k}=\int_{0}^{\infty} Q_{i}^{2}(x) d \psi^{*}(x) / x^{k}$ have been evaluated in terms of the constants $\pi_{n}, 1 / \lambda_{n} \pi_{n}$ in the appendix. For future references we record the values of $a_{1}$ and $a_{2}$ given by the theorem.

$$
\begin{aligned}
& a_{1}=\frac{1}{\rho q_{i} \pi_{i}} \\
& a_{2}=\frac{2}{\rho q_{i}^{2} \pi_{i}}+\frac{2}{q_{i} \pi_{i}}\left[\sum_{n=i}^{\infty} \frac{1}{\lambda_{n} \pi_{n}}\left(\sum_{j=n}^{\infty} \pi_{j+1}\right)^{2}+\sum_{n=0}^{i-1} \frac{1}{\lambda_{n} \pi_{n}}\left(\sum_{j=0}^{n} \pi_{j}\right)^{2}\right]
\end{aligned}
$$

(see formula (9.21)).

By a similar argument starting from the formula

$$
\int_{0}^{\infty} e^{-s t} d F_{i j}(t)=1-\frac{s}{\rho} \frac{\int_{0}^{\infty} \frac{1}{x+s}\left[Q_{j}^{2}(x)-Q_{i}(x) Q_{j}(x)\right] d \psi^{*}(x)}{1+\frac{1}{\rho} \int_{0}^{\infty} \frac{s}{x+s} Q_{j}^{2}(x) d \psi^{*}(x)}
$$

the moments of $F_{i j}$ can be computed:

THEOREM 5. In order that $\int_{0}^{\infty} t^{k+1} d F_{i j}(t)(i \neq j)$ be finite it is necessary and sufficient that $\int_{0}^{\infty} d \psi^{*} / x^{k}$ be finite, in which case

$$
b_{k+1}=\int_{0}^{\infty} t^{k+1} d F_{i j}(t)=\frac{(k+1) !}{\rho} \sum_{l=0}^{k} T_{l} c_{k-l} \quad(k \geqq 0)
$$

where 


$$
\begin{aligned}
c_{0} & =1 \\
c_{n} & =\sum_{\nu_{1}+2 \nu_{2}+\cdots+k v_{k}=n} \rho^{-\left(\nu_{1}+\cdots+\nu_{k}\right)} \frac{\left(\nu_{1}+\cdots+\nu_{k}\right) !}{\nu_{1} ! \cdots \nu_{k} !} I_{1}^{\nu_{1}} \cdots I_{k}^{\nu_{k}}, \\
I_{r} & =\int_{0}^{\infty} Q_{j}^{2}(x) \frac{d \psi^{*}(x)}{x^{r}}, \\
T_{r} & =\int_{0}^{\infty} \frac{Q_{j}^{2}(x)-Q_{i}(x) Q_{j}(x)}{x} \frac{d \psi^{*}(x)}{x^{r}} .
\end{aligned}
$$

We now turn to the study of the distributions $F_{i i}(t)$ for large $i$. The limiting behavior of these quantities as $i \rightarrow \infty$ has been studied by Bellman and Harris [4] and Harris [5]. The theorem below should be compared with Theorem 2 of [4], and Theorem 1 of [5].

It is assumed that $\sum \pi_{n}<\infty, \sum 1 / \lambda_{n} \pi_{n}=\infty$. If $N_{i i}$ is the time of first return to $i$ when the initial state is $i$ then $E\left(N_{i i}\right)=1 / \rho q_{i} \pi_{i}$ and

$$
G_{i}(t)=\operatorname{Pr}\left\{\frac{N_{i i}}{E\left(N_{i i}\right)} \leqq t\right\}=F_{i i}\left(\frac{t}{\rho q_{i} \pi_{i}}\right) .
$$

Hence from (3.2)

$$
1-\int_{0}^{\infty} e^{-s t} d G_{i}(\rho t)=\frac{1}{\left(1+\pi_{i} s\right)\left(\frac{\rho}{s}+q_{i} \pi_{i} \int_{0}^{\infty} Q_{i}^{2}(x) \frac{d \psi^{*}}{x+q_{i} \pi_{i} s}\right)}
$$

and it can be concluded that if for $s>0$

$$
q_{i} \pi_{i} \int_{0}^{\infty} Q_{i}^{2} \frac{d \psi^{*}}{x+q_{i} \pi_{i} s} \rightarrow c<\infty \quad \text { as } i \rightarrow \infty,
$$

then $G_{i}$ converges (in the sense of distributions) as $i \rightarrow \infty$ to the distribution $G(t ; c)$ whose Laplace transform is

$$
1-\frac{1}{1 / s+c}=\frac{c-1}{c}+\frac{1}{c} \cdot \frac{1 / c}{1 / c+s},
$$

i.e. to the distribution with a jump $(c-1) / c$ at $t=0$ and density $c^{-2} e^{-t / c}$ on $t>0$. The limit $c$, if it exists, is automatically $\geqq 1$, since for each $i$ and $s$ the left side of (3.7) is between 0 and 1 .

Theorem 6. If $\int_{0}^{\infty} d \psi^{*} / x<\infty$ and

$$
\lim _{i \rightarrow \infty} q_{i} \pi_{i} \sum_{k=0}^{i-1} \frac{1}{\lambda_{k} \pi_{k}}=c<\infty
$$

then 


$$
\lim _{i \rightarrow \infty} \operatorname{Pr}\left\{\frac{N_{i i}}{E\left(N_{i i}\right)} \leqq t\right\}=G(t ; c),
$$

Proof. Under the hypothesis it follows from equation (9.21) of the appendix that

$$
\lim _{i \rightarrow \infty} q_{i} \pi_{i} \int_{0}^{\infty} Q_{i}^{2} \frac{d \psi^{*}}{x}=c
$$

Thus it is sufficient to show that

$$
q_{i} \pi_{i}\left[\int_{0}^{\infty} Q_{i}^{2} \frac{d \psi^{*}}{x}-\int_{0}^{\infty} Q_{i}^{2} \frac{d \psi^{*}}{x+q_{i} \pi_{i} s}\right] \rightarrow 0
$$

as $i \rightarrow \infty$. This quantity can be written as (see (9.4))

$$
\begin{aligned}
\left(q_{i} \pi_{i}\right)^{2} s \int_{0}^{\infty} & Q_{i}^{2} \frac{d \psi^{*}}{x\left(x+q_{i} \pi_{i} s\right)}=\left(q_{i} \pi_{i}\right)^{2} s \int_{0}^{\infty} Q_{i} \frac{d \psi^{*}}{x\left(x+q_{i} \pi_{i} s\right)} \\
& -q_{i} \pi_{i} s \sum_{k=0}^{i-1} \frac{1}{\lambda_{k} \pi_{k}} \sum_{l=0}^{\min (M, k)} \pi_{l} q_{i} \pi_{i} \int_{0}^{\infty} Q_{i} Q_{l} \frac{d \psi^{*}}{x+q_{i} \pi_{i} s} \\
& -q_{i} \pi_{i} s \sum_{k=0}^{i-1} \frac{1}{\lambda_{k} \pi_{k}} \sum_{1+\min (M, k)}^{k} \pi_{l} q_{i} \pi_{i} \int_{0}^{\infty} Q_{i} Q_{l} \frac{d \psi^{*}}{x+q_{i} \pi_{i} s}
\end{aligned}
$$

where $M$ is a large fixed integer to be chosen. The three terms on the right of (3.8) will be denoted by $T_{1}, T_{2}$, and $T_{3}$ respectively. The divergence of $\sum 1 / \lambda_{n} \pi_{n}$ implies that $q_{i} \pi_{i} \rightarrow 0$, and since for all large $i$

$$
\left|q_{i} \pi_{i}-c / \sum_{0}^{i-1} \frac{1}{\lambda_{n} \pi_{n}}\right|<\frac{c}{2} / \sum_{0}^{i-1} \frac{1}{\lambda_{n} \pi_{n}}
$$

it is seen that

$$
\sup _{i} \max _{0 \leqq l \leqq i} \frac{q_{i} \pi_{i}}{q_{l} \pi_{l}} \leqq B_{1}<\infty .
$$

The integrals $q_{i} \pi_{i} \int_{0}^{\infty} Q_{i}^{2} d \psi^{*} / x$ are uniformly bounded, say $\leqq B_{2}$. The inequality

$$
\left|q_{i} \pi_{i} \int_{0}^{\infty} Q_{i} Q_{l} \frac{d \psi^{*}}{x+q_{i} \pi_{i} s}\right| \leqq\left(q_{i} \pi_{i} \int_{0}^{\infty} Q_{i}^{2} \frac{d \psi^{*}}{x}\right)^{1 / 2}\left(\frac{q_{i} \pi_{i}}{q_{l} \pi_{l}} q_{l} \pi_{l} \int_{0}^{\infty} Q_{l}^{2} \frac{d \psi^{*}}{x}\right)^{1 / 2}
$$

shows that the left side is $\leqq B_{1}^{1 / 2} B_{2}$ for $0 \leqq l \leqq i$ and for each fixed $l, \rightarrow 0$ as $i \rightarrow \infty$.

It follows at once that $T_{1} \rightarrow 0$ as $i \rightarrow \infty$. Given $\epsilon>0$ let $M$ be chosen so large that $\sum_{l=M+1}^{\infty} \pi_{l}<\epsilon$ and then choose $i$ so large that 


$$
\left|q_{i} \pi_{i} \int_{0}^{\infty} Q_{i} Q_{l} \frac{d \psi^{*}}{x+q_{i} \pi_{i} s}\right|<\epsilon \text { for } l=0,1, \cdots, M
$$

We then have

$$
\begin{aligned}
& \left|T_{2}\right|<\left(q_{i} \pi_{i} s \sum_{k=0}^{i-1} \frac{1}{\lambda_{k} \pi_{k}}\right) \sum_{l=0}^{\infty} \pi_{l} \cdot \epsilon, \\
& \left|T_{3}\right|<\left(q_{i} \pi_{i} s \sum_{k=0}^{i-1} \frac{1}{\lambda_{k} \pi_{k}}\right) \epsilon B_{1}^{1 / 2} B_{2},
\end{aligned}
$$

from which the result follows.

By imposing further assumptions on the rates of convergence of $q_{i} \pi_{i}$. $\sum_{j=1}^{i-1} 1 / \lambda_{j} \pi_{j}$ to $c$, it is possible to deduce with these methods results about the rate of convergence of the moments of the distribution of $N_{k k} / E\left(N_{k k}\right)$ to the moments of the limiting distribution.

If $\lambda_{n} / \mu_{n} \rightarrow a<1$, an easy calculation will show that the conditions of Theorem 6 are fulfilled with $c=1+a / 1-a$.

4. Transient processes $\left(\mu_{0}=0\right)$. The following criterion is an immediate consequence of Theorem 2 .

The birth and death process is transient if and only if $\sum \pi_{n}=\infty$ and $\sum 1 / \lambda_{n} \pi_{n}<\infty$.

In terms of the particle description, to say that the process is transient means that with positive probability (in fact, according to Theorem 9, with probability 1 ), the particle drifts to infinity. The point at infinity should be regarded as a permanent absorbing state which may be reached in finite or infinite time. Two types of transient processes can be distinguished, according to the rate at which the diffusing particle drifts to infinity. The transient process is called of type 1 if for some finite $t>0$, and for some $i$

$$
\sum_{j=0}^{\infty} P_{i j}(t)<1,
$$

and is called of type 2 if it is not of type 1 . If the process is of type 1, then it follows from the semi-group property that the inequality (4.1) is valid for every $i$ and for all $t>0$. For given $i$ and (finite) $t$

$$
\operatorname{Pr}\left\{\lim _{\tau \rightarrow t-0} X(\tau)=\infty \mid X(0)=i\right\}=1-\sum_{j=0}^{\infty} P_{i j}(t),
$$

which shows that the transient process is of type 1 or 2 according as the probability of reaching infinity in a given finite time is positive or zero. The next theorem provides an easy way of distinguishing between the two types of transient behavior.

THEOREM 7. If the process is transient the following conditions are equivalent: 
(i) the process is of type 2, that is $\sum_{j=0}^{\infty} P_{i j}(t)=1$ for all $i$ and all $t>0$,

$$
\sum_{n=0}^{\infty} \pi_{n} \sum_{i=n}^{\infty} \frac{1}{\lambda_{i} \pi_{i}}=\infty
$$

Proof. The equivalence of (i) and (ii) was proved in [1 (Theorem 16)].

The next theorem is trivial for processes of type 1 but gives some information about the path functions of type 2 processes.

THEOREM 8. For any transient process

$$
\operatorname{Pr}\left\{\lim _{t \rightarrow \infty} X(t)=\infty \mid X(0)=i\right\}=1 .
$$

Proof. The arbitrary initial state is fixed throughout the discussion. Let $E_{j}$ denote the set of all paths for which the particle spends an infinite amount of time in state $j$. Since the time spent in state $j$ on a particular visit has a negative exponential distribution with parameter $\lambda_{j}+\mu_{j}$, the set $E_{j}$ differs only by a set of measure zero from the set of all paths which visit $j$ infinitely often. If $\operatorname{Pr}\left\{E_{j}\right\}>0$ then the expected occupation time of state $j$ is infinite, i.e.

$$
\int_{0}^{\infty} P_{i j}(t) d t=\int_{0}^{\infty} Q_{i}(x) Q_{j}(x) \frac{d \psi}{x}=\infty,
$$

so that $\sum_{0}^{\infty} 1 / \lambda_{n} \pi_{n}=\infty$, and the process is recurrent, contrary to hypothesis. Hence $\operatorname{Pr}\left\{E_{j}\right\}=0$ and if $E=U_{j} E_{j}$ then $\operatorname{Pr}\{E\}=0$. If the path $X(t)$ is not in $E$ then clearly $X(t) \rightarrow \infty$ as $t \rightarrow \infty$, and hence

$$
\operatorname{Pr}\{X(t) \rightarrow \infty \mid X(0)=i\}=1 \text {. }
$$

Let $\bar{t}$ be the time at which the particle reaches infinity when the initial state is $i$. Then

$$
H_{i}(t) \equiv \operatorname{Pr}\{\bar{t} \leq t\}=1-\sum_{j=0}^{\infty} P_{i j}(t)
$$

For processes of type $2, \operatorname{Pr}\{\bar{t}=\infty\}=1$. For processes of type 1 we have the following result.

Theorem 9. If $\operatorname{Pr}\{\bar{t}<\infty\}>0$, then $\operatorname{Pr}\{\bar{t}<\infty\}=1$ and $1-H_{i}(t)$ tends exponentially to zero as $t \rightarrow \infty$.

Proof. The hypothesis implies that $1-\sum_{j=0}^{\infty} P_{i j}(t)>0$. By Theorem 7, $\sum_{0}^{\infty} \pi_{n} \sum_{k=n}^{\infty} 1 / \lambda_{k} \pi_{k}<\infty$ which implies that $-Q_{n}^{\prime}(0)$ remains bounded (see [1, Lemma 4]). By Theorem 16 of [1] the spectrum of $\psi$ begins at $a>0$ and concentrates at the zeros of $Q_{\infty}(x)=\lim _{n \rightarrow \infty} Q_{n}(x)$. If $0<b<a$, then $Q_{n}(b)$ $>Q_{\infty}(b)>0$ and by equation (4.13a) of [1] 


$$
\sum_{j=0}^{\infty} P_{i j}(t) \leqq \frac{e^{-b t}}{Q_{\infty}(b)} .
$$

This last equation shows that $1-H_{i}(t)$ tends exponentially to zero. The first part of the theorem is an immediate consequence of this fact.

It is of some interest to compute the moments of the distributions $H_{i}(t)$. For simplicity, let the initial state be zero.

$$
\begin{aligned}
E(\bar{t}) & =\int_{0}^{\infty} t d H_{0}(t)=-\int_{0}^{\infty} t \sum_{j=0}^{\infty} P_{0 j}^{\prime}(t) d t \\
& =\sum_{j=0}^{\infty} \pi_{j} \int_{0}^{\infty} \int_{0}^{\infty} x t e^{-x t} Q_{j}(x) d \psi(x) d t=\sum_{j=0}^{\infty} \pi_{j} \int_{0}^{\infty} \frac{Q_{j}(x)}{x} d \psi .
\end{aligned}
$$

By (9.9) we obtain

$$
E(\bar{t})=\sum_{j=0}^{\infty} \pi_{j} \sum_{n=j}^{\infty} \frac{1}{\lambda_{n} \pi_{n}} .
$$

The higher moments reduce to

$$
E\left(\bar{t}^{n}\right)=n ! \sum_{j=0}^{\infty} \pi_{j} \int_{0}^{\infty} \frac{Q_{j}}{x^{n}} d \psi .
$$

These integrals obviously exist since the spectrum of $\psi$ begins at $a>0$. With the aid of the formula (9.4) through (9.8), these can be expressed in terms of $\lambda_{n}$ and $\mu_{n}$. For example

$$
E\left(\bar{t}^{2}\right)=2 \sum_{j=0}^{\infty} \pi_{j} \sum_{m=j}^{\infty} \frac{1}{\lambda_{m} \pi_{m}} \sum_{i=0}^{m} \pi_{i} \sum_{k=i}^{\infty} \frac{1}{\lambda_{k} \pi_{k}} .
$$

An important problem associated with the study of stochastic processes is the question of the existence of a stationary measure. In the case of birth and death processes the problem takes the following form: When does there exist a non-negative nontrivial solution to the equation

$$
a_{i}=\sum_{j} a_{j} P_{j i}(t), \quad i=0,1,2, \cdots .
$$

It is not necessarily required that the series $\sum a_{i}$ be finite. For the case of recurrent Markov chains a solution was given by Derman [9] and later Chung [2] extended the same method to the continuous time recurrent Markov chain. Harris has recently dealt with the problem for the general discrete time recurrent Markov process. For birth and death processes we present a complete analysis which is independent of any recurrence assumptions. We start with the special case $\mu_{0}=0$. If $\sum_{i} P_{j i}(t)=1$ then $a_{j}=\pi_{j}$ is clearly a solution in view of the identity $\pi_{j} P_{j i}(t)=\pi_{i} P_{i j}(t)$. To establish uniqueness we use a device introduced by Derman. Suppose $a_{i} \geqq 0$ is a non- 
trivial solution of (4.3). Since $P_{j i}(t)>0$ for all $t>0$ it follows that $a_{i}>0$ and we may take $a_{0}=1$. Define

$$
q_{i j}(t)=\frac{a_{j}}{a_{i}} P_{j i}(t)
$$

It is readily seen that $q_{i j}(t)$ define transition probabilities of a birth and death process with parameters $\lambda_{i}^{\prime}=a_{i+1} \mu_{i+1} / a_{i}$ and $\mu_{i}^{\prime}=a_{i-1} \lambda_{i-1} / a_{i}, i \geqq 1$, which must satisfy the relations

$$
\lambda_{i}^{\prime}+\mu_{i}^{\prime}=\frac{a_{i+1}}{a_{i}} \mu_{i+1}+\frac{a_{i-1}}{a_{i}} \lambda_{i-1} \quad i \geqq 1
$$

where $\mu_{n}$ and $\lambda_{n}$ represent the parameters of the original process. We can verify inductively that the only solution of the equation (4.5) is $a_{i}=\pi_{i}$ which completes the proof of the uniqueness. We now demonstrate the converse proposition which is to the effect that if (4.3) has a non-negative solution $a_{i}$, which is necessarily positive, then $\sum_{j} P_{i j}(t) \equiv 1$. In fact, if $q_{i j}(t)$ is defined as in (4.4), then we deduce that $\sum q_{i j}(t) \equiv 1$ and again because of the relations (4.5) we find that $a_{i}=\pi_{i}$. It follows that $P_{i j}(t) \equiv q_{i j}(t)$ and so $\sum_{j} P_{i j}(t) \equiv 1$ obtains.

Combining the analysis with that of Theorem 7 we state the result as follows: A necessary and sufficient condition that (4.3) possess a positive solution is that $\sum_{j=0}^{\infty} \pi_{j} \sum_{k=j}^{\infty} 1 / \lambda_{k} \pi_{k}=\infty$. Furthermore, the only possible solution of (4.3) is necessarily a multiple of $\left\{\pi_{j}\right\}$.

In the case where $\mu_{0}>0$ we state the result without proof. If $\gamma$ represents the smallest positive value belonging to the spectrum of $\psi$ then $\left\{\pi_{j} Q_{j}(\gamma)\right\}$ is a solution to (4.3) if and only if $\sum_{j=0}^{\infty} \pi_{j} Q_{j}(\gamma)=\infty$. The solution when it exists is unique except for a multiplicative factor.

5. Absorption processes $\left(\mu_{0}>0\right)$. When $\mu_{0}>0$ it is necessary to consider in addition to the states $0,1,2, \cdots$ a state with index -1 . When the particle is in the 0 state and a transition occurs, the particle goes to 1 with probability $\lambda_{0} /\left(\lambda_{0}+\mu_{0}\right)$ and to -1 with probability $\mu_{0} /\left(\lambda_{0}+\mu_{0}\right)$. Once the -1 state has been reached the particle remains there ever afterward. The event of reaching -1 is called absorption at zero.

The polynomials in this case have the property that $Q_{n}(0)$ is strictly increasing. The conditions (i) $Q_{n}(0) \rightarrow \infty$, (ii) $\mu_{0} \int_{0}^{\infty} d \psi(x) / x=1$ and (iii) $\sum_{0}^{\infty} 1 / \lambda_{n} \pi_{n}$ $=\infty$ are equivalent (see [1, Lemma 6]).

For $i \geqq 0$ let $G_{i}(t)$ be the probability that absorption at zero occurs before time $t$ given that at $t=0$ the particle is in state $i$. Then

$$
G_{i}(t)=\mu_{0} \int_{0}^{t} P_{i 0}(\tau) \mathrm{d} \tau=\mu_{0} \int_{0}^{t} d \tau \int_{0}^{\infty} e^{-x \tau} Q_{i}(x) d \psi(x) .
$$


Using the results of $\S 9$, the following theorem is obtained.

THEOREM 10. If the initial state is $i$, the probability of eventual absorption at zero is

$$
\int_{0}^{\infty} d G_{i}(t)=\mu_{0} \int_{0}^{\infty} \frac{Q_{i}(x)}{x} d \psi(x)=1-\frac{Q_{i}(0)}{\lim _{n \rightarrow \infty} Q_{n}(0)}=\frac{\mu_{0} \sum_{n=i}^{\infty} \frac{1}{\lambda_{n} \pi_{n}}}{1+\mu_{0} \sum_{n=0}^{\infty} \frac{1}{\lambda_{n} \pi_{n}}} .
$$

This is 1 for every $i$ or $<1$ for every $i$ according as $\sum_{n=0}^{\infty} 1 / \lambda_{n} \pi_{n}$ diverges or converges. The formula

$$
\int_{0}^{\infty} t^{n} d G_{i}(t)=n ! \mu_{0} \int_{0}^{\infty} \frac{Q_{i}(x)}{x^{n+1}} d \psi(x)
$$

is valid whenever either integral exists. In particular (see (9.20))

$$
\int_{0}^{\infty} t d G_{i}(t)=\frac{1}{\mu_{0}} \sum_{k=0}^{\infty} \pi_{k}+\sum_{j=0}^{i-1} \frac{1}{\lambda_{j} \pi_{j}} \sum_{r=j+1}^{\infty} \pi_{r}
$$

whenever either member is finite.

Absorption at zero is called certain if the probability of eventual absorption at zero is 1 , and is called ergodic if it is certain and the expected time of absorption is finite. Absorption at 0 is called transient if it is not certain. These properties are independent of the initial state and from the preceding theorem it is seen that absorption at zero is

(i) certain if and only if $\sum 1 / \lambda_{n} \pi_{n}$ diverges,

(ii) ergodic if and only if $\sum 1 / \lambda_{n} \pi_{n}$ diverges and $\sum \pi_{n}$ converges.

When absorption at zero is not certain the particle eventually reaches infinity with probability $Q_{i}(0) / \lim _{n \rightarrow \infty} Q_{n}(0)$. In the case when absorption at zero is not certain, it is of some interest to determine conditions for the existence of the moments of the conditional distribution of absorption. In fact, the explicit evaluation of these moments can be accomplished. Assuming for simplicity that the initial state is zero the conditional distribution of absorption is

$$
\frac{\mu_{0} \int_{0}^{t} P_{00}(s) d s}{\mu_{0} \int_{0}^{\infty} P_{00}(s) d s}=\left[\frac{1+\mu_{0} \sum_{k=0}^{\infty} \frac{1}{\lambda_{k} \pi_{k}}}{\sum_{k=0}^{\infty} \frac{1}{\lambda_{k} \pi_{k}}}\right] \int_{0}^{\infty}\left[\frac{1-e^{-x t}}{x}\right] d \psi
$$

(see (9.12)). Of course, since absorption is not certain, the series $\sum 1 / \lambda_{k} \pi_{k}$ converges. The $r$ th moment, provided it exists, reduces to the form 


$$
r ! \int_{0}^{\infty} \frac{d \psi}{x^{r+1}} \frac{\left(1+\mu_{0} \sum_{k=0}^{\infty} \frac{1}{\lambda_{k} \pi_{k}}\right)}{\sum_{k=0}^{\infty} \frac{1}{\lambda_{k} \pi_{k}}}
$$

These integrals in turn can be expressed in terms of $\lambda_{n}$ and $\mu_{n}$ by means of the formulas (9.11) through (9.16). In particular, the expected length of time until absorption, given that absorption happens in finite time and the initial state of the particle is zero, is

$$
E\{A \mid A<\infty, X(0)=0\}=\frac{\sum_{j=0}^{\infty} \pi_{j}\left(\sum_{i=j}^{\infty} \frac{1}{\lambda_{i} \pi_{i}}\right)^{2}}{\left(1+\mu_{0} \sum_{i=0}^{\infty} \frac{1}{\lambda_{i} \pi_{i}}\right)\left(\sum_{k=0}^{\infty} \frac{1}{\lambda_{k} \pi_{k}}\right)} .
$$

(Here the random variable $A$ is the time at which absorption takes place.) If this last series converges, then the interpretation is that for those paths where absorption does take place, it must happen rather quickly.

When the -1 state is absorbing, in view of the result $\int d \psi / x<\infty$, the measure $\psi$ cannot possess mass at zero. Consequently, $\lim _{t \rightarrow \infty} P_{i j}(t)=0$ for every $i, j \geqq 0$. We now examine $\lim _{t \rightarrow \infty} \sum_{j=0}^{\infty} P_{i j}(t)$ for fixed $i$. If absorption ultimately occurs with certainty, then $\lim _{t \rightarrow \infty} \sum_{j=0}^{\infty} P_{i j}(t)={ }^{\prime}$. In fact

$$
\text { Prob }\{\text { absorption in time } \leqq t \mid X(0)=i\}+\sum_{j=0}^{\infty} P_{i j}(t) \leqq 1
$$

and the first term tends to one. Let us now assume that absorption is not certain or equivalently $\sum 1 / \lambda_{n} \pi_{n}<\infty$. The principle assertion is that equality in (5.2) is valid for every $t$ and every $i$ if and only if $\sum_{n=0}^{\infty} \pi_{n} \sum_{i=n}^{\infty} 1 / \lambda_{s} \pi_{i}$ $=\infty$. The proof is similar to that of Theorem 7 and is omitted. In fact, an appeal to probabilistic considerations will show that the above assertion can be reduced to the case of Theorem 7. Since the first term of (5.2) approaches monotonically a value less than 1 , we find that $\lim _{t \rightarrow \infty} \sum_{j=0}^{\infty} P_{i j}(t)=a>0$ is equivalent to the divergence of $\sum \pi_{n} \sum_{i=n}^{\infty} 1 / \lambda_{i} \pi_{i}$. If $\sum \pi_{n} \sum_{i=n}^{\infty} 1 / \lambda_{i} \pi_{i}<\infty$, then it can be shown that $\sum_{j=0}^{\infty} P_{i j}(t)$ converges exponentially to zero. (See Theorem 8.)

An interesting random variable associated with the absorption process (assuming absorption is certain) is the number $N$ of transitions which occur before absorption. The total number of transitions to the right which occur before absorption is a random variable $M$ related to $N$ and the initial state $i$ by

$$
N=i+2 M .
$$


The random variable $M$ was first considered by Kendall in connection with linear growth models [6].

Let

$$
R_{i}^{n}=\operatorname{Pr}\{N=n \mid X(0)=i\} ; \quad i=0,1,2, \cdots, n=1,2, \cdots .
$$

After the first transition the particle is at $i+1$ with probability $p_{i}$ and at $i-1$ with probability $q_{i}$ where

$$
p_{i}=\frac{\lambda_{i}}{\lambda_{i}+\mu_{i}}, \quad q_{i}=\frac{\mu_{i}}{\lambda_{i}+\mu_{i}} .
$$

Hence $R_{t}^{n}$ satisfies the recurrence relation

$$
R_{0}^{n}=p_{0} R_{1}^{n-1}, \quad R_{i}^{n}=q_{i} R_{i-1}^{n-1}+p_{i} R_{i+1}^{n-1},
$$

for $n>1$, and the initial condition

$$
R_{i}^{1}= \begin{cases}q_{0} & \text { if } i=0 \\ 0 & \text { if } i>0\end{cases}
$$

Let $S_{i}=\sum_{n=1}^{\infty} R_{i}^{n}$. Then $0 \leqq S_{i} \leqq 1$, and from the above relations

$$
S_{0}=p_{0} S_{1}+q_{0}, \quad S_{i}=q_{i} S_{i-1}+p_{i} S_{i+1},
$$

so

$$
S_{i+1}-S_{i}=\frac{q_{i}}{p_{i}}\left(S_{i}-S_{i-1}\right)=\frac{q_{0} \cdots q_{i}}{p_{0} \cdots p_{i}}\left(S_{0}-1\right)
$$

and hence

$$
S_{n+1}-1=\left(1+\mu_{0} \sum_{i=0}^{n} \frac{1}{\lambda_{i} \pi_{i}}\right)\left(S_{0}-1\right) .
$$

When $\sum_{n=0}^{\infty} 1 / \lambda_{i} \pi_{i}$ diverges this gives $S_{n}=1$ for all $n$. If $\sum_{n=0}^{\infty} 1 / \lambda_{n} \pi_{n}$ converges there is positive probability of never being absorbed at zero and hence $S_{n}<1$ for all $n$.

The recurrence relations

$$
\begin{aligned}
T_{0}(x) & \equiv 1, \\
x T_{0}(x) & =p_{0} T_{1}(x), \\
x T_{n}(x) & =q_{n} T_{n-1}(x)+p_{n} T_{n+1}(x), \quad n \geqq 1,
\end{aligned}
$$

determine a system of polynomials $T_{n}(x)$ orthogonal on the interval $-1 \leqq x \leqq 1$ with respect to a measure $d \alpha(x)$ of total mass 1 . To see this, first consider the polynomials $V_{n}(x)=T_{n}(1-x)$ which satisfy $V_{0}(x) \equiv 1$ and 


$$
\begin{aligned}
& -x V_{0}=-V_{0}+p_{0} V_{1}, \\
& -x V_{n}=q_{n} V_{n-1}-V_{n}+p_{n} V_{n+1},
\end{aligned}
$$

These polynomials are clearly the polynomials belonging to a birth and death process, and hence are orthogonal on $0 \leqq x<\infty$. Consequently $V_{n}(x)>0$ for $x \leqq 0$ and $T_{n}(x)>0$ for $x \geqq 1$. Since $T_{n}(x)$ is even or odd with $n$, all the zeros of the polynomials $T_{n}(x)$ are therefore in $-1<x<1$, and all the zeros of the $V_{n}(x)$ are in $0<x<2$. It follows that the polynomials $T_{n}$ are orthogonal on $-1 \leqq x \leqq 1$. The measure $\alpha$ is unique and in all cases of practical interest it can be computed explicitly.

It is easily verified that

$$
R_{i}^{n}=q_{0} \int_{-1}^{1} x^{n-1} T_{i}(x) d \alpha(x) .
$$

This formula may be viewed as expressing an absorption probability related to a random walk. The random walk takes place on the integers $0,1,2, \ldots$. When the particle is at $i$ it moves in the next step to $i+1$ with probability $p_{i}$ and to $i-1$ with probability $q_{i}$. When it is at 0 it moves in the next step to 1 with probability $p_{0}$ and is absorbed with probability $q_{0}$. The transition probability matrix of this random walk is

$$
P_{i j}^{n}=\pi_{j}^{*} \int_{-1}^{1} x^{n} T_{i}(x) T_{j}(x) d \alpha(x)
$$

where

$$
\pi_{n}^{*}=\pi_{n} \frac{\lambda_{n}+\mu_{n}}{\lambda_{0}+\mu_{0}} .
$$

If the particle starts at $i$ then the probability it is absorbed on the $n$th step is $R_{i}^{n}$. The random variable $M$ and $N$ are concerned only with the number of transitions which occur before absorption, and not with the speed at which these transitions occur; thus it is natural that they should lead to random walk problems.

As $k \rightarrow \infty, 1+2 x+\cdots+k x^{k-1}$ converges monotonically to $1 /(1-x)^{2}$ on $0 \leqq x \leqq 1$. Consequently

$$
\sum_{n=1}^{\infty} n R_{i}^{n}=q_{0} \int_{-1}^{1} \frac{1}{(1-x)^{2}} T_{i}(x) d \alpha(x)
$$

if either member is finite. Assuming that $\sum 1 / \lambda_{i} \pi_{i}$ diverges, $\sum_{n=1}^{\infty} n R_{i}^{n}$ is the expected number of steps until absorption, and the above formula shows that this is finite for all $i$ or for none, according as $\int_{-1}^{1} d \alpha(x) /(1-x)^{2}$ converges or diverges.

We assume that $\sum 1 / \lambda_{n} \pi_{n}$ diverges and hence for every $i$ 


$$
1=\sum_{n=1}^{\infty} R_{i}^{n}=q_{0} \int_{-1}^{1} \frac{1}{1-x} T_{i}(x) d \alpha(x) .
$$

Setting $i=0$ gives

$$
q_{0} \int_{-1}^{1} \frac{d \alpha(x)}{1-x}=1
$$

The polynomials $V_{n}(x)$ mentioned above are orthogonal on $0 \leqq x \leqq 2$ with respect to the measure $\beta$ defined by

$$
\beta(x)=+\int_{1-x}^{1} d \alpha(s)
$$

and hence

$$
q_{0} \int_{-1}^{1} \frac{d \alpha(x)}{(1-x)^{2}}=q_{0} \int_{0}^{2} \frac{d \beta(x)}{x^{2}}=q_{0} \int_{0}^{\infty} \frac{d \beta(x)}{x^{2}}
$$

and this integral is of a type already evaluated:

$$
q_{0} \int_{0}^{\infty} \frac{d \beta(x)}{x^{2}}=\frac{1}{q_{0}} \sum_{n=0}^{\infty} \pi_{n}^{*}
$$

equality holding if either member is finite. Consequently,

$$
q_{0} \int_{-1}^{1} \frac{1}{(1-x)^{2}} d \alpha(x)=\frac{1}{q_{0}} \sum_{n=0}^{\infty} \pi_{n}^{*}=\left(\frac{2}{\mu_{0}} \sum_{n=0}^{\infty} \lambda_{n} \pi_{n}+1\right) .
$$

Therefore, in order for $N$ to have a finite expected value it is necessary and sufficient that the series $\sum \lambda_{n} \pi_{n}$ converge. The higher moments can be analyzed in a similar manner.

6. Relations of processes with $\mu_{0}>0$ and $\mu_{0}=0$. Although the birth and death process where -1 is an absorbing state and the process where 0 is a reflecting barrier have been discussed separately, various useful relations exist between these two kinds of processes. Let $Q_{n}(x)$ be orthogonal polynomials, with respect to a measure $\psi$ which correspond to the reflecting barrier process (A) $\left(\mu_{0}=0\right)$ having birth and death rates, $\lambda_{n}(n \geqq 0)$ and $\mu_{n}(n>1)$ respectively. The functions $H_{n}(x)=\lambda_{n} \pi_{n}\left[Q_{n+1}(x)-Q_{n}(x)\right] /(-x)$ are a system of orthogonal polynomials with respect to the measure $x d \psi / \lambda_{0}$ and generate the process (B) with birth rates $\lambda_{n}^{*}$ and death rates $\mu_{n}^{*}$ where $\lambda_{n}^{*}=\mu_{n+1}$ and $\mu_{n}^{*}=\lambda_{n}$, valid for $n \geqq 0$. The process (B) possesses an absorbing state at -1 . The two processes (A) and (B) can be thought of as dual to each other in the sense that the role of the birth and death rates are interchanged combined with a shift of $\mu_{n}$. Since $1 / \lambda_{n}^{*} \pi_{n}^{*}=\pi_{n+1} / \lambda_{0}$ and $\pi_{n}^{*}=\lambda_{0} / \lambda_{n} \pi_{n}$, it follows that if process (A) is ergodic, recurrent null or transient then process (B) is respectively ergodic absorbing, certain absorbing, or transient absorbing. 
Suppose $T_{n}(x)$ are the orthogonal polynomials (with respect to a measure $\theta(x))$ obeying the recurrence law

$$
-x T_{n}(x)=-\left(\lambda_{n}+\mu_{n}\right) T_{n}(x)+\lambda_{n} T_{n+1}(x)+\mu_{n} T_{n-1}(x) \quad n \geqq 0
$$

where $\mu_{0}>0$ and $T_{-1}(x) \equiv 0$. The corresponding process (C) has -1 as an absorbing state. The polynomials $S_{n+1}(x)=\left(\lambda_{n} \pi_{n} / \lambda_{0}\right)\left[T_{n+1}(x)-T_{n}(x)\right], S_{0}(x)$ $=1$ constitute an orthogonal system with respect to the measure

$$
\left\{\begin{array}{cc}
\mu_{0} \frac{d \theta(x)}{x} \quad \text { for } x>0 ; \\
1-\mu_{0} \int \frac{d \theta(x)}{x} \text { for } x=0 .
\end{array}\right.
$$

The process (D) generated by the polynomials $S_{n}(x)$ has the state 0 as a reflecting barrier. The birth and death rates $\lambda_{n}^{*}$ and $\mu_{n}^{*}$ respectively of the process (D) satisfy the relations $\lambda_{n}^{*}=\mu_{n}(n \geqq 0)$ and $\mu_{n}^{*}=\lambda_{n-1}(n \geqq 1)$ where $\mu_{n}$ and $\lambda_{n}$ are the parameters appearing in (6.1). Process (C) bears the same relation to process (D) as process (B) bears to process (A).

Another set of polynomials closely related to $Q_{n}(x)$ are the associated system $Q_{n}^{(0)}$ defined as

$$
Q_{n}^{(0)}(x)=\int_{0}^{\infty} \frac{Q_{n}(x)-Q_{n}(t)}{x-t} d \psi(t) .
$$

Observe that $Q_{n}^{(0)}(x)$ is of degree $n-1$ and $Q_{1}^{(0)}(x) \equiv-1 / \lambda_{0}$. It is known that $-\lambda_{0} Q_{n}^{(0)}(x)=W_{n-1}(x)$ satisfy the recursion formula

$$
-x W_{n-1}=-\left(\lambda_{n}+\mu_{n}\right) W_{n-1}+\lambda_{n} W_{n}+\mu_{n} W_{n-2}
$$

for all $n \geqq 1$ where $W_{0} \equiv 1$ and $W_{-1} \equiv 0$. The $W_{n}$ form a system of orthogonal polynomials with respect to a measure $\alpha$ on $0 \leqq x<\infty$. A method for computing $\alpha$ when $\psi$ is known, is given in $\S 8$. The usefulness and significance of the $W_{n}$ is that a representation of the first passage time distributions $F_{i 0}(t)$ in terms of the $W_{n}$ can be given. In fact, the $W_{n}$-process is obtained from the original process by stopping it whenever the zero state is reached. Thus for $i \geqq 1$, the first passage time probability $F_{i 0}(t)$ of the original process is the same as the probability of absorption before time $t$ with initial state $i-1$, for the $W_{n}$-process:

$$
F_{i 0}(t)=\mu_{1} \int_{0}^{t} d \tau \int_{0}^{\infty} e^{-x \tau} W_{i-1}(x) d \alpha(x) .
$$

In a similar way the first passage time distributions $F_{i k}(t), i>k$, can be expressed in terms of the $k$ th associated system $Q_{n}^{(k)}(x)$ defined by 


$$
Q_{n}^{(k)}(x)=\pi_{k} \int_{0}^{\infty} Q_{k}(y) \frac{Q_{n}(x)-Q_{n}(y)}{x-y} d \psi(y) .
$$

In determining the first passage time distribution $F_{0 i}(i>0)$, we convert state $i$ into an absorbing state by truncating the matrix $A$ as shown:

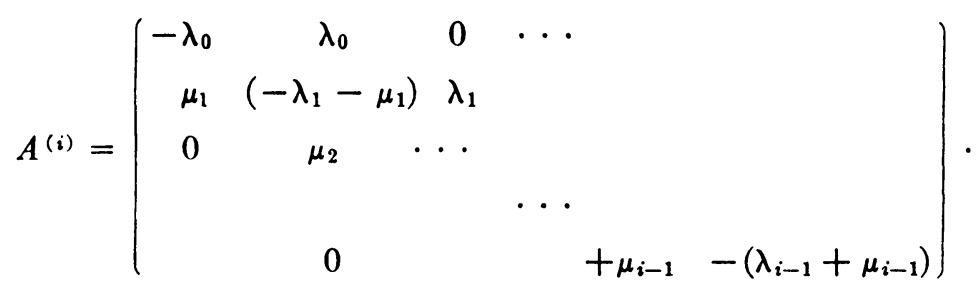

This defines a process with a finite number of states for which

$$
\lambda_{i-1} /\left(\mu_{i-1}+\lambda_{i-1}\right)
$$

is the rate of absorption from the $(i-1)$ th state into the permanent absorbing state $i$. In this case only a finite set of polynomials is determined by the vector relation $A^{(i)} Q=-x Q$. They agree with $Q_{j}(x)$ for $0 \leqq j \leqq i-1$. The corresponding measure $\psi^{(i)}$ is discrete and its spectrum consists of the zeros of the polynomial $-x Q_{i-1}+\left(\lambda_{i-1}+\mu_{i-1}\right) Q_{i-1}-\mu_{i-1} Q_{i-2}$. The probability of absorption in time $\leqq t$ into state $i$ from state 0 is easily seen to be

$$
\lambda_{i-1} \pi_{i-1} \int_{0}^{t} d s \int_{0}^{\infty} e^{-x s} Q_{i-1}(x) d \psi^{(i)}(x) .
$$

This is of course the same as the first passage time distribution $F_{0 i}(t)$ for the original unrestricted birth and death process.

Next suppose it is required to compute the probability that absorption at zero occurs before time $t$ and without the state $i$ ever having been visited, assuming the initial state is $j, 0 \leqq j \leqq i-1$. Denoting this probability by $i A_{j}(t)$, we have

$$
{ }_{i} A_{j}(t)=\mu_{0} \int_{0}^{\infty} \frac{1-e^{-x t}}{x} Q_{j}(x) d \psi^{(i)}(x)
$$

where $\psi^{(i)}$ is the distribution introduced above. In particular the probability of eventual absorption at zero without the state $i$ ever being visited, when the initial state is $j$, is

$$
{ }_{i} A_{j}=\mu_{0} \int_{0}^{\infty} Q_{j}(x) \frac{d \psi^{(i)}(x)}{x} .
$$

Now since $\psi^{(i)}$ concentrates on the zeros of $Q_{i}(x)$

$$
Q_{i}^{(j)}(y)=\pi_{j} \int_{0}^{\infty} Q_{j}(x) \frac{Q_{i}(y)-Q_{i}(x)}{y-x} d \psi^{(i)}(x)=Q_{i}(y) \pi_{j} \int_{0}^{\infty} Q_{j}(x) \frac{d \psi^{(i)}(x)}{1-x}
$$


and setting $y=0$ gives

$$
{ }_{i} A_{j}=-\mu_{0} \frac{Q_{i}^{(j)}(0)}{Q_{i}(0) \pi_{j}} .
$$

Now the polynomials $Q_{i}^{(j)}(x)$ satisfy [1]

$$
\begin{aligned}
& -x Q_{i}^{(j)}(x)=\mu_{i} Q_{i-1}^{(j)}(x)-\left(\lambda_{i}+\mu_{i}\right) Q_{i}^{(j)}(x)+\lambda_{i} Q_{i+1}^{(j)}(x), \\
& i>j, Q_{j}^{(j)}(x) \equiv 0, Q_{j+1}^{(j)} \equiv-\frac{1}{\lambda_{j}}
\end{aligned}
$$

and by a simple computation for $i>j$,

$$
-Q_{i}^{(j)}(0)=\frac{1}{\lambda_{j} \pi_{j}}\left[1+\lambda_{j} \pi_{j} \sum_{k=j+1}^{i-1} \frac{1}{\lambda_{k} \pi_{k}}\right] .
$$

Consequently,

$$
{ }_{i} A_{j}=\mu_{0} \sum_{k=j}^{i-1} \frac{1}{\lambda_{k} \pi_{k}} /\left(1+\mu_{0} \sum_{k=0}^{i-1} \frac{1}{\lambda_{k} \pi_{k}}\right) .
$$

A result similar to this has previously been obtained by Harris [5, Theorem 2b].

We are also able to obtain explicit expressions for $E\left(N_{10}^{r} \mid N_{10}<\infty\right)$ whenever finite, where the random variable $N_{10}$ is the length of time until the particle first enters state 0 given the initial state is 1 . Of course, the results are new only when the process is transient. The conditions for recurrence were fully investigated in $\S 1$. Introducing the measure $\alpha(x)$ corresponding to polynomial system $Q^{(0)}(x)$, the zero state becomes an absorbing state, and the original random variable $N_{10}$ is identified with the random variable $A$ describing the time up to absorption. Consequently,

$$
E\left(N_{10}^{r} \mid N_{10}<\infty\right)=E\left[A^{r} \mid A<\infty, X(0)=1\right] .
$$

Appealing to (9.16) where these latter moments have been computed, we obtain in particular

$$
\operatorname{Exp}\left(N_{10} \mid N_{10}<\infty\right)=\int \frac{d \alpha(x)}{x^{2}} / \int \frac{d \alpha}{x} \quad \text { (see equation (5.1)) }
$$

Since $\lambda_{n}^{*}, \mu_{n}^{*}$ for the new process in terms of $\lambda_{n}, \mu_{n}$ for the original process are $\lambda_{n}^{*}=\lambda_{n+1}$ and $\mu_{n}^{*}=\mu_{n+1}$, by (9.9) we get

$$
\operatorname{Exp}\left(N_{10} \mid N_{10}<\infty\right)=\frac{\sum_{i=1}^{\infty} \pi_{j}\left(\sum_{i=j}^{\infty} \frac{1}{\lambda_{i} \pi_{i}}\right)^{2}}{\left(1+\lambda_{0} \sum_{i=1}^{\infty} \frac{1}{\lambda_{i} \pi_{i}}\right)\left(\sum_{i=1}^{\infty} \frac{1}{\lambda_{i} \pi_{i}}\right)} .
$$


Finally, it is interesting to observe that the problem of determining, for a recurrent process, the distribution of the number of transitions which occur before the expiration of a first passage time or a first return time, also leads to consideration of the random walk embedded in the birth and death process. For example suppose we have a recurrent process $\left(\mu_{0}=0, \sum_{0}^{\infty} 1 / \lambda_{n} \pi_{n}=\infty\right)$, and the random variable $Z$ is the number of transitions which occur before the first return to zero, the initial state being zero. Then $N=Z-1$ is the number of transitions which occur before absorption for a random walk on the integers $1,2,3, \cdots$. Using the expected value for $N$ computed in $\S 5$, it is found that

$$
E(Z)=\frac{1}{\lambda_{0}} \sum_{n=0}^{\infty} \pi_{n}\left(\lambda_{n}+\mu_{n}\right) .
$$

This is finite or infinite according as $\sum \lambda_{n} \pi_{n}$ converges or diverges.

The example $\lambda_{n}=n+1, \mu_{0}=0, \mu_{n}=(n+1)^{2} / n$ for $n \geqq 1$, shows that there are ergodic processes for which $E(Z)=\infty$, while on the other hand the example $\lambda_{n}=1 /(n+1), \mu_{0}=0, \mu_{n}=(n+1) / n^{2}$ for $n \geqq 1$, shows that there are recurrent null processes for which $E(Z)$ is finite.

7. Ratio theorems $\left(\mu_{0} \geqq 0\right)$. A remarkable property of birth and death processes, obvious from the integral representation, is the fact that

$$
\frac{P_{i j}(t)}{P_{j i}(t)}=\frac{\pi_{j}}{\pi_{i}}
$$

is independent of $t$.

The classical Doeblin ratio theorem asserts that $\left(^{(1)}\right.$

$$
\lim _{t \rightarrow \infty} \frac{\int_{0}^{t} P_{i j}(t) d t}{\int_{0}^{t} P_{k l}(t) d t}
$$

is finite and positive. The integral representation makes it easy to prove a much deeper theorem. The question of investigating the ratio was suggested to the authors by T. Harris.

TheOREM 11. $\lim _{t \rightarrow \infty} P_{i j}(t) / P_{k l}(t)$ exists and is finite and positive.

Proof. Let $a \geqq 0$ be the smallest point in the support of $\psi$. From the theory of orthogonal polynomials it is known that $Q_{n}(a)>0$ for every $n$. Hence

$$
P_{i j}(t)=e^{-a t} \pi_{j} Q_{i}(a) Q_{j}(a) \int_{a}^{\infty} e^{-(x-a) t} f(x) d \psi(x)
$$

(1) Doeblin discussed only the discrete time case. The continuous time case was first analyzed by K. L. Chung [2]. 
where

$$
f(x)=\frac{Q_{i}(x) Q_{j}(x)}{Q_{i}(a) Q_{j}(a)} .
$$

We study the ratio

$$
\frac{\int_{a}^{\infty} e^{-(x-a) t} f(x) d \psi(x)}{\int_{a}^{\infty} e^{-(x-a) t} d \psi(x)}=1+\frac{\int_{a}^{\infty} e^{-(x-a) t}[f(x)-1] d \psi(x)}{\int_{a}^{\infty} e^{-(x-a) t} d \psi(x)}=1+R .
$$

Now $f$ is continuous and $f(a)=1$ so given $\epsilon$ there is a $\delta>0$ such that $|f(x)-1|$ $<\epsilon$ for $a \leqq x \leqq a+\delta$. Hence

$$
\begin{aligned}
|R| & \leqq \frac{\left|\int_{a}^{a+\delta}\right|+\left|\int_{a+\delta}^{\infty}\right|}{\int_{a}^{\infty} e^{-(x-a) t} d \psi(x)} \leqq \epsilon+\frac{\int_{a+\delta}^{\infty} e^{-(x-a) t}|f(x)-1| d \psi(x)}{\int_{a}^{a+\delta / 2} e^{-(x-a) t} d \psi(x)} \\
& \leqq \epsilon+c e^{\delta t / 2} \int_{a+\delta}^{\infty} e^{-(x-a) t}|f(x)-1| d \psi(x)
\end{aligned}
$$

where $c=\left[\int_{a}^{a+\delta / 2} d \psi(x)\right]^{-1}$ which is finite and positive. It follows that $\lim \sup _{t \rightarrow \infty}|R| \leqq \epsilon$ and therefore $R \rightarrow 0$ as $t \rightarrow \infty$. From this the theorem follows at once. In fact we see that

$$
\lim _{t \rightarrow \infty} \frac{P_{i j}(t)}{P_{k l}(t)}=\frac{\pi_{j} Q_{i}(a) Q_{j}(a)}{\pi_{l} Q_{k}(a) Q_{l}(a)}
$$

If $a=0$ and $\mu_{0}=0$, then

$$
\lim _{t \rightarrow \infty} \frac{P_{i j}(t)}{P_{k l}(t)}=\frac{\pi_{j}}{\pi_{l}}
$$

The Doeblin ratio theorem is an abelian form of the above theorem in the recurrent case and follows from it easily. In the ergodic case $\left(\mu_{0}=0\right)$ $P_{i j}(t) \rightarrow \pi_{j} \rho$ so $t^{-1} \int_{0}^{t} P_{i j}(\tau) d \tau \rightarrow \pi_{j} \rho$ and hence

$$
\lim _{t \rightarrow \infty} \frac{\int_{0}^{t} P_{i j}(\tau) d \tau}{\int_{0}^{t} P_{k l}(\tau) d \tau}=\frac{\pi_{j}}{\pi_{l}}=\lim _{t \rightarrow \infty} \frac{P_{i j}(t)}{P_{k l}(t)} .
$$

In the recurrent null case $\left(\mu_{0}=0\right), a=0$ by Theorem 1 , so $P_{i j}(t) / P_{k l}(t) \rightarrow \pi_{j} / \pi_{l}$ while $\int_{0}^{\infty} P_{i j}(t) d t$ diverges, and by a standard abelian argument the Doeblin 
ratio also $\rightarrow \pi_{j} / \pi_{l}$. In the transient case the limits of the two ratios may be different. We compute the Doeblin ratio limits explicitly, assuming $\mu_{0}=0$. In this case

$$
\sum_{0}^{\infty} \frac{1}{\lambda_{n} \pi_{n}}=\int_{0}^{\infty} \frac{d \psi}{x}
$$

is finite and

$$
\int_{0}^{\infty} P_{i j}(t) d t=\pi_{j} \int_{0}^{\infty} \frac{Q_{i}(x) Q_{j}(x)}{x} d \psi(x)
$$

If $i \leqq j$ then (appendix, formulas (9.9) and (9.10))

$$
\int_{0}^{\infty} P_{i j}(t) d t=\pi_{j} \int_{0}^{\infty} \frac{Q_{j}(x)}{x} d \psi(x)=\pi_{j} \sum_{n=j}^{\infty} \frac{1}{\lambda_{n} \pi_{n}}
$$

and hence for $i \leqq j, k \leqq l$

$$
\lim _{t \rightarrow \infty} \frac{\int_{0}^{t} P_{i j}(\tau) d \tau}{\int_{0}^{t} P_{k l}(\tau) d \tau}=\frac{\pi_{j} \sum_{n=j}^{\infty} \frac{1}{\lambda_{n} \pi_{n}}}{\pi_{l} \sum_{n=l}^{\infty} \frac{1}{\lambda_{n} \pi_{n}}} .
$$

On the other hand

$$
\lim _{t \rightarrow \infty} \frac{P_{i j}(t)}{P_{k l}(t)}
$$

is $\pi_{j} / \pi_{l}$ if the support of $\psi$ reaches to zero, and depends on $i$ and $k$ otherwise.

8. The computation of $\psi \cdot\left(\mu_{0} \geqq 0\right)$. Almost all the models of birth and death processes that have been discussed in the literature are associated with a system of polynomials $\left\{Q_{n}(x)\right\}$, which, after some simple change of variable and renormalization, are classical polynomials. For these processes the computation of the distribution $\psi$ presents no problem. However there are important special processes for which the corresponding polynomials do not reduce to classical polynomials. In this section we discuss a method of computing $\psi$ when the recurrence formulas, that is the constants $\lambda_{n}, \mu_{n}$, are given.

The function

$$
B(s)=\int_{0}^{\infty} \frac{d \psi(x)}{x+s}
$$

is an analytic function of the complex variable $s$, regular in the sector $|\arg s|<\pi,|s|>0$. The Stieltjes inversion formula 


$$
\int_{0}^{x} d \psi(y)=\frac{1}{\pi} \lim _{\eta \rightarrow 0+} \int_{-\epsilon}^{x} \operatorname{Im} B(-\xi-i \eta) d \xi
$$

expresses the measure $\psi$ in terms of $B$. It is valid when $\epsilon>0$ and $x$ is a point of continuity of $\int_{0}^{x} d \psi(y)$. Consequently the problem of computing $\psi$ is reduced to the problem of finding $B(s)$. If

$$
R_{i j}(s)=\int_{0}^{\infty} e^{-s t} P_{i j}(t) d t
$$

then

$$
R_{j 0}(s)=Q_{j}^{(0)}(-s)+B(s) Q_{j}(-s)
$$

and

$$
R_{0 j}(s)=Q_{j}^{(0)}(-s) \pi_{j}+B(s) Q_{j}(-s) \pi_{j} .
$$

There are two cases to consider. First, if $\sum_{0}^{\infty} 1 / \lambda_{n} \pi_{n}$ diverges, or more generally if $\sum_{0}^{\infty} 1 / \lambda_{n} \pi_{n} \sum_{0}^{n} \pi_{i}$ diverges then for fixed $s>0$ both $Q_{j}(-s)$ and $Q_{j}^{\prime 0)}(-s)$ are unbounded as $j \rightarrow \infty$. (See Lemma 4 of [1].) In this case it is seen from (8.3) that $B(s)$ is uniquely determined by the condition that, for fixed $s>0, R_{j 0}(s)$ is bounded as $j \rightarrow \infty$. In the second case, when $\sum \pi_{n}$ diverges, both of the series $\sum_{j} Q_{j}(-s) \pi_{j}$ and $\sum_{j} Q_{j}^{(0)}(-s) \pi_{j}$ diverge for all $s>0$, and it follows from (8.4) that $B(s)$ is uniquely determined by the condition that $\sum_{j=0}^{\infty} R_{0 j}(s)$ converges for $s>0$.

In either of these cases, if the asymptotic behavior of the polynomials $Q_{n}(x)$ and $Q_{n}^{\prime \prime)}(x)$ for $n$ large and $x$ in an interval $-\alpha \leqq x \leqq-\beta$ on the negative real axis is known, then $B(s)$ can be determined by the above conditions for $\beta \leqq s \leqq \alpha$. In practice $B(s)$ usually turns out to be a relatively simple function, and the problems of continuing it in to the complex plane, and of applying the inversion formula, present only minor technical difficulties. A number of important examples will be treated in a separate publication.

The necessary and sufficient condition in order that the solution of the Hamburger moment problem generated by the polynomials $Q_{n}(x)$ be unique is that the series

$$
\sum_{n=1}^{\infty} \pi_{n}\left(\sum_{k=0}^{n-1} \frac{1}{\lambda_{k} \pi_{k}}\right)^{2}
$$

diverges (a proof can be based on [7, Theorem 2.17]). In practice this condition is usually satisfied, and when it is the function $B(s)$ is uniquely determined for nonreal $s$ by the requirement

$$
\sum_{n=0}^{\infty}\left|Q_{n}^{(0)}(-s)+B(s) Q_{n}(-s)\right|^{2} \pi_{n}<\infty .
$$


We now turn to the computation of the measure $\alpha$ belonging to the associated polynomials $Q_{n}^{(0)}(x)$. Let $F_{00}(t)$ be the probability that a particle which starts at the zero state returns to zero for the first time before time $t$ after having left zero. This function is not in general a probability distribution. If

$$
\hat{F}_{00}(s)=\int_{0}^{\infty} e^{-s t} d F_{00}(t),
$$

then

$$
\hat{F}_{00}(s)=1-\frac{1}{\lambda_{0}+\mu_{0}+s}\left(\int_{0}^{\infty} \frac{d \psi}{x+s}\right)^{-1}, \quad s>0(\text { see (1.3)) }
$$

Let

$$
\widetilde{P}_{i j}(t)=\operatorname{Pr}\{X(t)=j, X(\tau) \neq 0 \text { for } 0 \leqq \tau \leqq t \mid X(0)=i\}
$$

for $i, j \geqq 1, t \geqq 0$. Then $\left(\tilde{P}_{i j}(t)\right)$ is the transition probability matrix of a birth and death process on the states $1,2,3, \ldots$ whose infinitesimal matrix is obtained from $A$ by deleting the first row and first column (see §8). The corresponding polynomials are $\left\{-\lambda_{0} Q_{n}^{(0)}(x)\right\}, n \geqq 1$, and

$$
\widetilde{P}_{i j}(t)=\frac{\mu_{1}}{\lambda_{0}} \pi_{j} \int_{0}^{\infty} e^{-x t}\left[-\lambda_{0} Q_{i}^{(0)}(x)\right]\left[-\lambda_{0} Q_{j}^{(0)}(x)\right] d \alpha(x)
$$

where $\alpha$ is a positive measure of total mass one, with respect to which the polynomials $Q_{n}^{(0)}(x)$ are orthogonal. From the relation

$$
F_{00}(t)=\frac{\lambda_{0}}{\lambda_{0}+\mu_{0}} \int_{0}^{t}\left(\lambda_{0}+\mu_{0}\right) e^{-\left(\lambda_{0}+\mu_{0}\right) r} \int_{0}^{t-r} \mu_{1} \widetilde{P}_{11}(\sigma) d \sigma
$$

it follows that

$$
\hat{F}_{00}(s)=\frac{\lambda_{0 \mu_{1}}}{\lambda_{0}+\mu_{0}+s} \int_{0}^{\infty} \frac{d \alpha(x)}{x+s} .
$$

Combining (8.5) and (8.6) gives

$$
\begin{aligned}
\int_{0}^{\infty} \frac{d \alpha(x)}{x+s} & =\frac{1}{\lambda_{0} \mu_{1}}\left[\lambda_{0}+\mu_{0}+s-1 / \int_{0}^{\infty} \frac{d \psi(x)}{x+s}\right] \\
& =\frac{1}{\lambda_{0} \mu_{1}}\left[\lambda_{0}+\mu_{0}+s-\frac{1}{B(s)}\right] .
\end{aligned}
$$

Hence by the inversion formula

$$
\int_{0}^{x} d \alpha(y)=\frac{1}{\lambda_{0} \mu_{1} \pi} \lim _{\eta \rightarrow 0+} \int_{-\epsilon}^{x} \frac{\operatorname{Im} B(-\xi-i \eta)}{|B(-\xi-i \eta)|^{2}} d \xi .
$$


Suppose $\psi$ is a discrete distribution with masses $\rho_{0}, \rho_{1}, \rho_{2}, \cdots$ located at the points $0 \leqq x_{0}<x_{1}<x_{2}<\cdots<x_{n} \rightarrow \infty$. Then

$$
B(-s)=\sum_{0}^{\infty} \frac{\rho_{n}}{x_{n}-s}
$$

is a meromorphic function whose only poles are simple poles at the points $x_{n}$. In each of the open intervals $x_{n}<s<x_{n+1}, B(-s)$ increases steadily from $-\infty$ to $+\infty$ and hence has exactly one zero $y_{n}$ in the interval. The function

$$
\int_{0}^{\infty} \frac{d \alpha(x)}{x+s}=\frac{1}{\lambda_{0} \mu_{1}}\left[\lambda_{0}+\mu_{0}+s-\frac{1}{B(s)}\right]
$$

is therefore also a meromorphic function, whose only poles are at the zeros $y_{n}$ of $B(-s)$. These poles are simple poles because, since $\operatorname{Im} B(-s)<0$ for Im $s>0$, the zeros of $B(-s)$ are all simple. Thus $\alpha$ is also a discrete distributiou whose jumps $y_{n}$ are at the zeros of $B(-s)$ and are interlocked with the jumps of $\psi$ :

$$
0 \leqq x_{0}<y_{0}<x_{1}<y_{1}<x_{2}<y_{2}<\cdots .
$$

The mass concentrated by $\alpha$ at $y_{n}$ is

$$
\gamma_{n}=-\frac{1}{B^{\prime}\left(-y_{n}\right)} \frac{1}{\lambda_{0} \mu_{1}} .
$$

For future reference we cite the following facts about the inversion of the Stieltjes transform in relation to the identity (8.7) which connects the measure $\psi$ and the measure $\alpha$.

Proposition A. If $\psi$ has a continuous positive density $\psi^{\prime}$ in an interval $a<x<b$, then in any closed subinterval $a<a^{\prime} \leqq x \leqq b^{\prime}<b$,

$$
\frac{1}{\lambda_{0} \mu_{1} \pi^{2}} \operatorname{Im} B(-\xi-i \eta) /|B(-\xi-i \eta)|^{2}
$$

converges boundedly to $\alpha^{\prime}(\xi)$ and

$$
\alpha^{\prime}(\xi)=\frac{1}{\lambda_{0} \mu_{1} \pi^{2}} \frac{\downarrow^{\prime}(\xi)}{\left(P . V \cdot \frac{1}{\pi} \int \frac{\psi^{\prime}(x)}{(x-\xi)} d x\right)^{2}+\left[\psi^{\prime}(\xi)\right]^{2}}
$$

(P.V. denotes principal value).

Proof. Consider

$$
\frac{1}{\pi} \operatorname{Im} B(-\xi-i \eta)=\frac{1}{\pi} \int_{a}^{b} \frac{\eta \psi^{\prime}(\xi)}{(x-\xi)^{2}+\eta^{2}} d \xi+O(\eta),
$$


for $\xi$ in $\left(a^{\prime}, b^{\prime}\right)$. The integral is recognizable as the Poisson transform applied to $\psi^{\prime}(\xi)$. The Poisson kernel for the half plane generates a regular summation method so that $\operatorname{Im} B(-\xi-i \eta) / \pi$ approaches $\psi^{\prime}(\xi)$ uniformly for $a^{\prime} \leqq \xi \leqq b^{\prime}$ as $\eta \rightarrow 0+$. The convergence is uniform as a consequence of the fact that $\psi^{\prime}(\xi)$ is continuous on the interval $a^{\prime} \leqq \xi \leqq b^{\prime}$ [8, see p. 31]. The study of the behavior of $1 /|B(-\xi-i \eta)|^{2}$ is deeper. Some further requirements are imposed on $\psi^{\prime}(\xi)$.

If $\psi^{\prime}(\xi)$ is continuous and in addition positive for $a^{\prime} \leqq \xi \leqq b^{\prime}$, then

$$
\frac{1}{|B(-\xi-i \eta)|^{2}} \leqq \frac{1}{(\operatorname{Im} B(-\xi-i \eta))^{2}} \rightarrow \frac{1}{\pi^{2} \psi^{\prime}(\xi)^{2}}
$$

the convergence being uniform so that

$$
\frac{1}{|B(-\xi-i \eta)|^{2}} \leqq C\left(a^{\prime}, b^{\prime}, \gamma\right)
$$

(the constant $C$ depending only on $a^{\prime}, b^{\prime}$ and $\gamma=\min _{a^{\prime} \leq \xi \leq b^{\prime}} \psi^{\prime}(\xi)$ ). Since $\psi^{\prime}(\xi)$ belongs to $L(0, \infty)$ it is a known result $\left[8\right.$, p. 132] that $\lim _{\eta \rightarrow 0+} \operatorname{Re} B(-\xi-i \eta)$ exists almost everywhere. Consequently, $\pi \operatorname{Im} B(-\xi-i \eta) /|B(-\xi-i \eta)|^{2}$ approaches boundedly a.e. the limit

$$
\frac{\psi^{\prime}(\xi)}{\left(\mathrm{P} . \mathrm{V} \cdot \frac{1}{\pi} \int \frac{\psi^{\prime}(x)}{x-\xi} d x\right)^{2}+\left[\psi^{\prime}(\xi)\right]^{2}}=\theta(\xi)
$$

([8, p. 132]) for $a^{\prime} \leqq \xi \leqq b^{\prime}$. Hence, on this same interval $\alpha^{\prime}(\xi)$ exists and is equal to $\left(1 / \lambda_{0} \mu_{1} \pi^{2}\right) \theta(\xi)$.

Proposition B. If a is the smallest point in the support of the measure $\psi$, then the smallest point in the support of $\alpha$ is $\geqq a$. Furthermore, $\alpha$ has no mass at $x=a$.

Proof. The first part follows immediately from the fact that $\int d \psi /(x+s)$ is analytic, real and of one sign for $s>-a$. Suppose that $x=a$ is a mass point for $\alpha$. Then $\lim _{s \rightarrow-a+0}(a+s) \int d \alpha /(x+s)=\lambda>0$ where $\lambda$ is the mass of $\alpha$ located at $a$. But,

$$
\lim _{s \rightarrow-a+0} \frac{(a+s)}{\lambda_{0} \mu_{1}}\left[\lambda_{0}+\mu_{0}+s-\frac{1}{B(s)}\right]=0
$$

since $B(y-a)=\int_{a}^{\infty} d \psi(x) /(x-a+y)$ is strictly positive and increases monotonically as $y \rightarrow 0+$ to a positive ( $\infty$ is a possible value) limit. On multiplying (8.7) by $a+s$ and allowing $s$ to approach $-a$ a contradiction results. The proof of the proposition is complete.

As an illustration of the above theory let $d \psi(x)=c e^{-x} x^{\beta} d x,-1<\beta<0$, then 


$$
\text { P.V. } \int_{0}^{\infty} \frac{d \psi(x)}{x-y}=-c(\pi \cot \pi \beta) e^{-y} y^{\beta}-c \Gamma(\beta+1) e^{-y} \int_{0}^{1} e^{y t} t^{-\beta-1}
$$

so that by Propositions A and B and (8.7), the measure $d \alpha$ is completely specified. The ideas of this section and this last example will be utilized in connection with the study of linear growth and queuing models to be published elsewhere.

9. ApPENDIX. Evaluation of integrals. The integrals $\int\left(Q_{i} Q_{j} / x^{n}\right) d \psi$, $\int\left(Q_{i} Q_{j} / x^{n}\right) d \psi^{*}$ will now be evaluated in terms of the constants $\lambda_{k}, \mu_{k}$. The computations make use of properties of the polynomials

$$
H_{n+1}(x)=\lambda_{n} \pi_{n}\left[Q_{n+1}(x)-Q_{n}(x)\right], \quad H_{0}(x)=\mu_{0} .
$$

These polynomials satisfy

$$
\begin{aligned}
-x H_{0}(x) & =-\mu_{0} H_{0}(x)+\mu_{0} H_{1}(x), \\
-x H_{n+1}(x) & =\lambda_{n} H_{n}(x)-\left(\lambda_{n}+\mu_{n+1}\right) H_{n+1}(x)+\mu_{n+1} H_{n+2}(x), \quad n \geqq 0 .
\end{aligned}
$$

The next two lemmas are proved in [1].

Lemma A. If $\mu_{0}>0$ there is a positive measure $\theta$ on $0 \leqq x<\infty$ such that

$$
\int_{0}^{\infty} \frac{H_{m}(x)}{\mu_{0}} \frac{H_{n}(x)}{\mu_{0}} d \theta(x)=\frac{\delta_{m n}}{\pi_{m}^{\prime}} \quad m, n=0,1,2, \cdots,
$$

where $\pi_{0}^{\prime}=1, \pi_{n}^{\prime}=\mu_{0} / \lambda_{n-1} \pi_{n-1}$ for $n \geqq 1$. If $\theta$ is such a measure then

$$
d \psi=x d \theta / \mu_{0}
$$

defines a solution $\psi$ of the moment problem belonging to the polynomials $\left\{Q_{n}\right\}$, for which

$$
\mu_{0} \int_{0}^{\infty} \frac{d \psi}{x} \leqq 1
$$

Conversely if $\psi$ is a solution of the $\left\{Q_{n}\right\}$ moment problem for which (9.3) is valid then the measure $\theta$ which has mass $1-\mu_{0} \int_{0}^{\infty}(d \psi / x)$ at $x=0$ and is defined on $0<x<\infty$ by $d \theta=\mu_{0} d \psi / x$, satisfies (9.1).

LeмmA B. If $\mu_{0}=0$ there is a positive measure $\theta$ on $0 \leqq x<\infty$ such that

$$
\int_{0}^{\infty} \frac{H_{m+1}(x)}{-x} \frac{H_{n+1}(x)}{-x} d \theta(x)=\frac{\delta_{m n}}{\pi_{n}^{\prime \prime}}, \quad m, n=0,1,2, \cdots,
$$

where $\pi_{0}^{\prime \prime}=1, \pi_{n}^{\prime \prime}=\lambda_{0} / \lambda_{n} \pi_{n}$ for $n \geqq 1$, and such that

$$
\lambda_{0} \int_{0}^{\infty} \frac{d \theta(x)}{x} \leqq 1 .
$$

If $\psi$ is any solution of the $\left\{Q_{n}\right\}$ moment problem then $d \theta(x)=x d \psi(x) / \lambda_{0}$ defines 
a measure $\theta$ with the above properties. Conversely if $\theta$ is such a measure and $\psi$ is the measure with mass $1-\lambda_{0} \int_{0}^{\infty} d \theta / x$ at $x=0$ and $d \psi(x)=\lambda_{0} d \theta(x) / x$ on $0<x<\infty$, then $\psi$ is a solution of the $\left\{Q_{n}\right\}$ moment problem.

The following lemma will also be needed.

Lemma C. If $\sum 1 / \lambda_{n} \pi_{n}<\infty$, and the solution of the moment problem is unique or equivalently $\sum \pi_{n}=\infty$, then $\int_{0}^{\infty} d \psi(x) / x^{k}<\infty$ implies

$$
\lim _{n \rightarrow \infty} \int_{0}^{\infty} \frac{Q_{n}(x)}{x^{k}} d \psi(x)=0 .
$$

Proof. The polynomials $Q_{0}^{*}(x)=Q_{n}(x) / Q_{n}(0)$ belong to a process with transition matrix $P_{i j}^{*}(t)=Q_{i}(0) / Q_{j}(0) P_{i j}(t)$, and with $\mu_{0}^{*}=0, \pi_{i}^{*}=Q_{i}^{2}(0) \pi_{i}$. It follows from [1, Theorem 5] that (1) for each $t>0, P_{j 0}^{*}(t)$ is monotone decreasing in $j$ and tends to a limit $\alpha(t) \geqq 0$ as $j \rightarrow \infty$, and (2) $\sum_{0}^{\infty} P_{0 j}^{*}(t) \leqq 1$. Consequently, since $P_{0 j}^{*}(t)=P_{j 0}^{*}(t) \pi_{j}^{*}$

$$
\sum_{j=0}^{\infty} P_{j 0}^{*}(t) \pi_{j}^{*} \leqq 1
$$

and since $\pi_{j}^{*} \geqq \pi_{j}, \sum \pi_{j}^{*}=\infty$, from which it follows that $\alpha(t) \equiv 0$. Thus for $t \geqq 0, P_{t 0}^{*}(t)$ converges monotonely to zero as $i \rightarrow \infty$. Now if

$$
\int_{0}^{\infty} \frac{d \psi}{x^{k}}<\infty
$$

then the integral

$$
\iint_{0 \leq \tau_{1} \leq \tau_{2} \leq \cdots \leq \tau_{k}<\infty} \cdots \int P_{i 0}^{*}\left(\tau_{k}\right) d \tau_{k} d \tau_{k-1} \cdots d \tau_{1}=\frac{1}{Q_{i}(0)} \int_{0}^{\infty} Q_{i}(x) \frac{d \psi}{x^{k}}
$$

is absolutely convergent and hence $\rightarrow 0$ as $i \rightarrow \infty$. Since

$$
1 \leqq Q_{i}(0) \leqq 1+\mu_{0} \sum_{0}^{\infty} \frac{1}{\lambda_{n} \pi_{n}}<\infty
$$

the lemma follows.

A. The integrals $\int_{0}^{\infty}\left(Q_{i} Q_{j} / x^{k}\right) d \psi$ when $\mu_{0}=0, \sum \pi_{n}=\infty$.

For given $k$ these integrals are either all convergent or all divergent. If $\int_{0}^{\infty} d \psi / x^{k}<\infty$, for some $k \geqq 1$, then $\sum_{0}^{\infty} 1 / \lambda_{n} \pi_{n}=\int_{0}^{\infty} d \psi / x<\infty$ and Lemma $C$ is applicable. From the identity

$$
Q_{m+1}(x)=1-x \sum_{n=0}^{m} \frac{1}{\lambda_{n} \pi_{n}} \sum_{i=0}^{n} \pi_{i} Q_{i}(x)
$$

we get 


$$
\int_{0}^{\infty} Q_{m+1}(x) \frac{d \psi}{x^{k}}=\int_{0}^{\infty} \frac{d \psi}{x^{k}}-\sum_{n=0}^{m} \frac{1}{\lambda_{n} \pi_{n}} \sum_{i=0}^{n} \pi_{i} \int_{0}^{\infty} Q_{i}(x) \frac{d \psi}{x^{k-1}}
$$

and letting $m \rightarrow \infty$

$$
\int_{0}^{\infty} \frac{d \psi}{x^{k}}=\sum_{n=0}^{\infty} \frac{1}{\lambda_{n} \pi_{n}} \sum_{i=0}^{n} \pi_{i} \int_{0}^{\infty} Q_{i}(x) \frac{d \psi}{x^{k-1}} .
$$

Combining (9.5) and (9.6)

$$
\int_{0}^{\infty} Q_{m}(x) \frac{d \psi}{x^{k}}=\sum_{n=m}^{\infty} \frac{1}{\lambda_{n} \pi_{n}} \sum_{i=0}^{n} \pi_{i} \int_{0}^{\infty} Q_{i}(x) \frac{d \psi}{x^{k-1}} .
$$

Multiplying (9.4) by $Q_{j}(x) / x^{k}$ and integrating gives

$$
\begin{aligned}
\int_{0}^{\infty} Q_{m}(x) Q_{j}(x) \frac{d \psi}{x^{k}} & \\
& =\int_{0}^{\infty} Q_{j}(x) \frac{d \psi}{x^{k}}-\sum_{n=0}^{m-1} \frac{1}{\lambda_{n} \pi_{n}} \sum_{i=0}^{n} \pi_{i} \int_{0}^{\infty} Q_{i}(x) Q_{j}(x) \frac{d \psi}{x^{k-1}} .
\end{aligned}
$$

The last two equations determine the integrals $\int_{0}^{\infty} Q_{i} Q_{j} d \psi / x^{k}$ in terms of the integrals $\int_{0}^{\infty} Q_{i} Q_{j} d \psi / x^{k-1}$. In particular

$$
\begin{array}{rlrl}
\int_{0}^{\infty} Q_{m}(x) \frac{d \psi}{x} & =\sum_{n=m}^{\infty} \frac{1}{\lambda_{n} \pi_{n}}, \\
\int_{0}^{\infty} Q_{m}(x) Q_{n}(x) \frac{d \psi}{x} & =\int_{0}^{\infty} Q_{m}(x) \frac{d \psi}{x} & \text { if } n \leqq m .
\end{array}
$$

B. The integrals $\int_{0}^{\infty} Q_{i} Q_{j} d \psi / x^{k}$ when $\mu_{0}>0, \sum 1 / \lambda_{n} \pi_{n}<\infty, \sum \pi_{n}=\infty$.

In this case $\int_{0}^{\infty} d \psi / x$ is convergent and if for some $k \geqq 1$

$$
\int_{0}^{\infty} \frac{d \psi}{x^{k}}<\infty
$$

then from Lemma $C$ and the identity

$$
\frac{Q_{n+1}(x)}{x^{k}}=\left(1+\mu_{0} \sum_{0}^{n} \frac{1}{\lambda_{i} \pi_{i}}\right) \frac{1}{x^{k}}-\sum_{0}^{n} \frac{1}{\lambda_{i} \pi_{i}} \sum_{0}^{i} \pi_{j} \frac{Q_{j}(x)}{x^{k-1}}
$$

we get

$$
\int^{\infty} \frac{d \psi}{x^{k}}=\frac{\sum_{0}^{\infty} \frac{1}{\lambda_{i} \pi_{i}} \sum_{0}^{i} \pi_{j} \int_{0}^{\infty} \frac{Q_{j}(x)}{x^{k-1}} d \psi}{Q_{\infty}(0)}
$$

where 


$$
Q_{\infty}(0)=1+\mu_{0} \sum_{0}^{\infty} \frac{1}{\lambda_{i} \pi_{i}}
$$

Using the identity again

$$
\begin{aligned}
\int_{0}^{\infty} Q_{n}(x) Q_{j}(x) \frac{d \psi}{x^{k}}= & \left(1+\mu_{0} \sum_{0}^{n-1} \frac{1}{\lambda_{i} \pi_{i}}\right) \int_{0}^{\infty} Q_{j}(x) \frac{d \psi}{x^{k}} \\
& -\sum_{0}^{n-1} \frac{1}{\lambda_{i} \pi_{i}} \sum_{0}^{i} \pi_{l} \int_{0}^{\infty} Q_{l}(x) Q_{j}(x) \frac{d \psi}{x^{k-1}} .
\end{aligned}
$$

These formulas determine the integrals recursively. In particular

$$
\begin{array}{rlrl}
\int_{0}^{\infty} Q_{n}(x) \frac{d \psi}{x} & =\frac{1}{Q_{\infty}(0)} \sum_{i=n}^{\infty} \frac{1}{\lambda_{i} \pi_{i}}, & n \geqq 0, \\
\int_{0}^{\infty} Q_{m}(x) Q_{n}(x) \frac{d \psi}{x}= & Q_{m}(0) \int_{0}^{\infty} Q_{n}(x) \frac{d \psi}{x}, & 0 \leqq m \leqq n<\infty, \\
\int_{0}^{\infty} Q_{n}(x) \frac{d \psi}{x^{2}}= & \frac{Q_{n}(0)}{Q_{\infty}^{2}(0)} \sum_{i=n}^{\infty} \frac{1}{\lambda_{i} \pi_{i}} \sum_{j=0}^{i} \pi_{j} \sum_{l=j}^{\infty} \frac{1}{\lambda_{l} \pi_{l}} \\
& -\frac{Q_{\infty}(0)-Q_{n}(0)}{Q_{\infty}^{2}(0)} \sum_{i=0}^{n-1} \frac{1}{\lambda_{i} \pi_{i}} \sum_{j=0}^{i} \pi_{j} \sum_{l=j}^{\infty} \frac{1}{\lambda_{l} \pi_{l}} .
\end{array}
$$

C. The integrals $\int_{0}^{\infty} Q_{i} Q_{j} d \psi / x^{k}$ when $\mu_{0}>0, \sum 1 / \lambda_{n} \pi_{n}=\infty$.

In this case it can be seen from the probability interpretation ( $\$ 5)$ that

$$
\mu_{0} \int_{0}^{\infty} \frac{d \psi}{x}=1
$$

This result can also be obtained from the identity

$$
\int_{0}^{\infty} \frac{d \psi}{x+s}=\sum_{0}^{\infty} \frac{1}{\lambda_{n} \pi_{n} Q_{n}(-s) Q_{n+1}(-s)}, \quad s \geqq 0,
$$

and the relations $\lambda_{n} \pi_{n}\left[Q_{n+1}(0)-Q_{n}(0)\right]=\mu_{0}, Q_{n}(0) \rightarrow \infty$. The polynomials $H_{n}(x) / \mu_{0}$ are an orthogonal system on $0 \leqq x<\infty$ with respect to the measure $d \theta=\mu_{0} d \psi / x$. The associated constants are $\pi_{n}^{\prime}=\mu_{0} / \lambda_{n-1} \pi_{n-1}$ for $n \geqq 1,1 / \lambda_{n}^{\prime} \pi_{n}^{\prime}$ $=\pi_{n} / \mu_{0}$, and $\mu_{0}^{\prime}=0$. Since $\sum \pi_{n}{ }^{\prime}=\infty$, this system is of the type treated in part $A$, and integrals of the form

$$
\int_{0}^{\infty} \frac{H_{m}(x)}{\mu_{0}} \frac{H_{n}(x)}{\mu_{0}} \frac{d \theta}{x^{k}}
$$

can be evaluated. In particular from the case $m=k=0, n \geqq 1$ it follows that 


$$
\mu_{0} \int_{0}^{\infty} Q_{n}(x) \frac{d \psi}{x}=1, \quad n \geqq 0
$$

From

$$
\int_{0}^{\infty} \frac{d \theta}{x}=\sum_{0}^{\infty} \frac{1}{\lambda_{n}^{\prime} \pi_{n}^{\prime}}
$$

follows

$$
\int_{0}^{\infty} \frac{d \psi}{x^{2}}=\frac{1}{\mu_{0}^{2}} \sum_{0}^{\infty} \pi_{n}
$$

The equations

$$
\int_{0}^{\infty} \frac{H_{n}(x)}{\mu_{0}} \frac{d \theta}{x^{k}}=\sum_{i=n}^{\infty} \frac{1}{\lambda_{i}^{\prime} \pi_{i}^{\prime}} \sum_{j=0}^{i} \pi_{j}^{\prime} \int_{0}^{\infty} \frac{H_{j}(x)}{\mu_{0}} \frac{d \theta}{x^{k-1}}
$$

and

$$
\begin{aligned}
\int_{0}^{\infty} \frac{H_{m}(x)}{\mu_{0}} \frac{H_{n}(x)}{\mu_{0}} & \frac{d \theta}{x^{k}} \\
= & \int_{0}^{\infty} \frac{H_{n}(x)}{\mu_{0}} \frac{d \theta}{x^{k}}-\sum_{i=0}^{m-1} \frac{1}{\lambda_{i}^{\prime} \pi_{i}^{\prime}} \sum_{j=0}^{i} \pi_{j}^{\prime} \int_{0}^{\infty} \frac{H_{j}(x)}{\mu_{0}} \frac{H_{n}(x)}{\mu_{0}} \frac{d \theta}{x^{k-1}}
\end{aligned}
$$

together with the identity

$$
Q_{n}(x)=1+\sum_{i=0}^{n-1} \frac{1}{\lambda_{i} \pi_{i}} H_{i+1}(x)
$$

determine the integrals recursively. It is found that

$$
\int_{0}^{\infty} Q_{n}(x) \frac{d \psi}{x^{2}}=\frac{1}{\mu_{0}^{2}} \sum_{0}^{\infty} \pi_{i}+\frac{1}{\mu_{0}} \sum_{0}^{n-1} \frac{1}{\lambda_{i} \pi_{i}} \sum_{i+1}^{\infty} \pi_{j} .
$$

D. The integrals $\int_{0}^{\infty} Q_{i} Q_{j} d \psi^{*} / x^{k}$.

When $\mu_{0}=0, \sum \pi_{n}<\infty, \sum 1 / \lambda_{n} \pi_{n}=\infty$, it is required to evaluate integrals of the above form, $\psi^{*}$ being the measure obtained from $\psi$ by removing the mass at the origin. In this case the polynomials $H_{n+1}(x) /-x$ are orthogonal with respect to the measure $d \theta=x d \psi / \lambda_{0}$. The integrals in question can be evaluated by making use of the formal identity

$$
\int_{0}^{\infty} f(x) \frac{d \psi^{*}}{x^{k}}=\lambda_{0} \int_{0}^{\infty} f(x) \frac{d \theta}{x^{k+1}}
$$

The constants associated with $\theta$ are $\mu_{0}^{\prime \prime}=\lambda_{0}, \pi_{n}^{\prime \prime}=\lambda_{0} / \lambda_{n} \pi_{n}, 1 / \lambda_{n}^{\prime \prime} \pi_{n}^{\prime \prime}=\pi_{n+1} / \lambda_{0}$. 
Since $\sum \pi_{n}^{\prime \prime}=\infty, \sum 1 / \lambda_{n}^{\prime \prime} \pi_{n}^{\prime \prime}<\infty, \mu_{0}^{\prime \prime}>0$, we are dealing with a situation of type B. In particular it is found that

$$
\int_{0}^{\infty} Q_{n}^{2}(x) \frac{d \psi^{*}}{x}=\frac{\sum_{i=n}^{\infty} \frac{1}{\lambda_{i} \pi_{i}}\left(\sum_{j=i}^{\infty} \pi_{j+1}\right)^{2}+\sum_{i=0}^{n-1} \frac{1}{\lambda_{i} \pi_{i}}\left(\sum_{j=0}^{i} \pi_{j}\right)^{2}}{\left(\sum_{k=0}^{\infty} \pi_{k}\right)^{2}} .
$$

\section{REFERENCES}

1. S. Karlin and J. L. McGregor, The differential equations of birth-and-death-processes and the Stieltjes moment problem, Trans. Amer. Math. Soc. vol. 85 (1957) pp. 489-546.

2. K. L. Chung, Foundations of the theory of continuous parameter Markoff chains, Proceedings of the Third Berkeley Symposium, vol. 2, 1956, pp. 29-40.

3. F. G. Foster, On the stochastic matrices associated with certain queuing processes, Ann. Math. Stat. vol. 24 (1953) pp. 355-360.

4. R. Bellman and T. E. Harris, Recurrence times for the Ehrenfest model, Pacific Journal of Mathematics vol. 1 (1951) pp. 184-187.

5. T. E. Harris, First passage and recurrence distributions, Trans. Amer. Math. Soc. vol. 73 (1952) pp. 471-486.

6. D. G. Kendall, On the generalized birth and death processses, Ann. Math. Stat. vol. 19 (1948) pp. 1-16.

7. J. A. Shohat and J. D. Tamarkin, The problem of moments, Mathematical Surveys, vol. 1, 1943.

8. E. C. Titchmarsh, Introduction to the theory of Fourier integrals, Oxford, 1948.

9. C. Derman, $A$ solution to a set of fundamental equations in Markov chains, Proc. Amer. Math. Soc. (1954) pp. 332-334.

California Institute of Technology, Pasadena, Calif. 